\title{
ON SURFACE SINGULARITIES OF MULTIPLICITY THREE*
}

\author{
JUN LU ${ }^{\dagger}$ AND SHENG-LI TAN ${ }^{\dagger}$
}

\author{
Dedicated to Prof. Stephen S.-T. Yau on his 60th birthday
}

\begin{abstract}
Let $P$ be a normal singularity of multiplicity $d=2$ or 3 of a complex surface $X$. It is well-known that $X$ is locally an irreducible finite cover $\pi: X \rightarrow Y$ of degree $d$ over a smooth surface $Y$, and the singularity $(X, P)$ can be resolved by the canonical resolution $X_{k} \rightarrow X_{k-1} \rightarrow \cdots \rightarrow X_{0}=X$, which is the pullback of the embedded resolution of the corresponding singularity $p=\pi(P)$ of the branch locus. Let $F$ be the maximal ideal cycle of this resolution. We will prove that $F$ has a unique decomposition $F=Z_{1}+\cdots+Z_{d}$ with $Z_{1} \geq Z_{2} \geq \cdots \geq Z_{d} \geq 0$, where $Z_{i}$ is a fundamental cycle or zero. We show that $w=p_{a}\left(Z_{1}\right)+\cdots+p_{a}\left(Z_{d}\right)$ is an invariant of $(X, P)$ that can also be computed from the multiplicity of the branch locus at $p .(X, P)$ is a rational singularity iff all of the singular points in the canonical resolution satisfies $w \leq d-1$. In order to get the minimal resolution from the canonical one, we need to blow down some exceptional curves, the number of blowing-downs is exactly that of fundamental cycles $Z$ in the canonical resolution satisfying $p_{a}(Z)=0$ and $Z^{2}=-1$.
\end{abstract}

Key words. Jung's resolution, canonical resolution, fundamental cycle, surface singularity, triple cover.

AMS subject classifications. Primary 14E15; Secondary 14B05, 32S45.

1. Introduction. Fundamental cycle (defined by M. Artin [2]), maximal ideal cycle (defined by S. S.-T. Yau [17]) and canonical cycle of a resolution are important invariants of a surface singularity. It is well-known that a surface singular point of multiplicity 2 or 3 admits a canonical resolution. Therefore, we get a sequence of maximal ideal cycles and fundamental cycles. Our first purpose is to compute explicitly the maximal ideal cycle of the canonical resolution. Then we will prove that each maximal ideal cycle can be decomposed as a sum of fundamental cycles. We use the sequence of fundamental cycles to define a sequence of numerical invariants. As an application, we will give a new criterion for the singularity to be rational. In order to get the minimal resolution from the canonical resolution, we need to blow down some $(-1)$-exceptional curves. We will prove that the number of curves blown down in the exceptional set is equal to that of the $(-1)$-fundamental cycles in the sequence. The main idea is to try to classify surface singularities by their branch loci.

There are several equivalent definitions of the multiplicity mult $P(X)$ of a singular point $P$ of $X$. We recall one of them (see [6], p.22). mult $_{P}(X)$ is the minimal degree of all finite local covers $\pi: X \rightarrow Y$ over a smooth local surface $Y$.

$$
m_{P}=\operatorname{mult}_{P}(X)=\min \{\operatorname{deg} \pi \mid \pi: X \rightarrow Y \text { is finite }\}
$$

The classical method is to present $(X, P)$ as a finite cover $\pi: X \rightarrow Y$ of degree $d=d_{P}$ over an open set $(Y, p)$ of $\mathbb{C}^{2}$ at the origin $p$ such that $\pi^{-1}(p)=P$. Then $p$ must be a singular point of the branch locus $B_{\pi}$ of $\pi$ in $Y$. The most useful resolution

*Received April 30, 2013; accepted for publication September 12, 2013. This work is supported by NSFC, the Science Foundation of the EMC and the Foundation of Scientific Program of Shanghai. The first author is also supported partly by the Fundamental Research Funds for the Central Universities.

$\dagger$ Department of Mathematics, and Laboratory of Pure Mathematics and Mathematical Practice, East China Normal University, Dongchuan RD 500, Shanghai 200241, P. R. of China (\{jlu; sltan\}@math.ecnu.edu.cn). 
of a normal surface singularity $(X, P)$ is the Jung's resolution as follows.

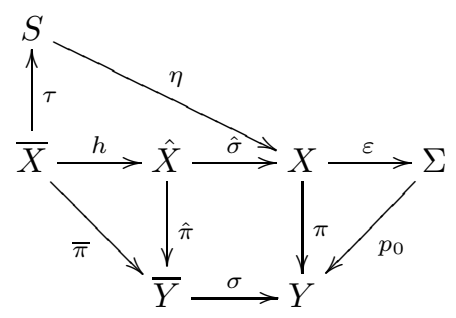

By a sequence of the blowing-ups $\sigma: \bar{Y} \rightarrow Y$ of $Y$ at the singular points of the branch locus, the pullback of $B_{\pi}$ in $\bar{Y}$ is a normal crossing divisor. The normalization $\hat{X}$ of $X \times_{Y} \bar{Y}$ is a finite cover $\hat{\pi}: \hat{X} \rightarrow \bar{Y}$ whose branch locus $B_{\hat{\pi}}$ is a normal crossing divisor contained in the pullback of $B_{\pi}$ in $\bar{Y}$. Then $\hat{X}$ admits at worst Hirzebruch-Jung singularities which can be resolved directly by the method of Hirzebruch, $h: \bar{X} \rightarrow \hat{X}$. Finally, we get the resolution morphism $\bar{\sigma}:=\hat{\sigma} \circ h: \bar{X} \rightarrow X$ which is called Jung's resolution of the singular point $(X, P)$ (see [10]). $\tau$ is the contraction of the $(-1)$ curves in the exceptional set of $\bar{\sigma}$, we get the minimal resolution $\eta: S \rightarrow X$.

By Noether's Normalization Theorem, $X$ is the normal model of a local surface $\Sigma \subset \mathbb{C}^{3}$ defined by an equation of degree $d$,

$$
z^{d}+a_{1}(x, y) z^{d-1}+\cdots+a_{d-1}(x, y) z+a_{d}(x, y)=0 .
$$

$\pi$ is the composition of the normalization map $\varepsilon: X \rightarrow \Sigma$ with the projection $p_{0}$ : $\Sigma \rightarrow Y$.

The maximal ideal cycle $M$ of a resolution $\bar{\sigma}:\left(\bar{X}, E_{P}\right) \rightarrow(X, P)$ is defined as the greatest divisor contained in any divisor of type $\operatorname{div}\left(\bar{\sigma}^{*} g\right)$, where $g$ is any nonzero holomorphic function on $X$ with $g(P)=0$. Namely,

$$
M=\operatorname{gcd}\left\{\operatorname{div}\left(\bar{\sigma}^{*} g\right) \mid 0 \neq g \in m_{P} \subseteq \mathcal{O}_{X, P}\right\} .
$$

If $d=2$ or 3 , then there exists $\sigma=\sigma_{0} \circ \cdots \circ \sigma_{k-1}$ such that the branch locus of $\hat{\pi}$ is a smooth curve, thus $\hat{X}$ is a smooth surface, i.e., $\bar{X}=\hat{X}$. This fact is proved by Horikawa for $d=2$, by Ashikaga [1] for hypersurface triple singularities, and by the second author for the general case with $d=3[12,13]$. This resolution $\bar{\sigma}: \bar{X}=\hat{X} \rightarrow X$ of $(X, P)$ is usually called the canonical resolution. We explain in detail the process of the canonical resolution.

Let $P_{0}=P$ and $p_{0}=p=\pi(P)$.

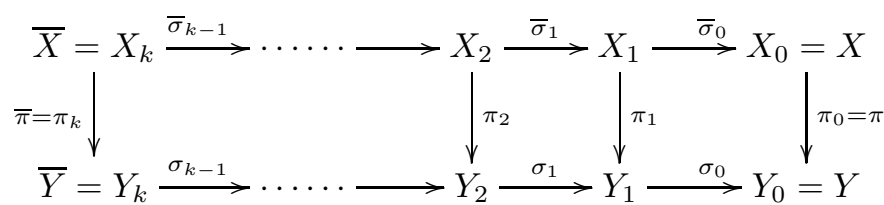

We know that $p_{0}$ is a singular point of the branch locus $B_{\pi_{0}}$. Let $\sigma_{0}: Y_{1} \rightarrow Y_{0}$ be the blowing-up of $Y_{0}$ at $p_{0}$, let $X_{1}$ be the normalization of $X_{0} \times_{Y_{0}} Y_{1}$, and let $\bar{\sigma}_{0}$ and $\pi_{1}$ be the induced morphisms. If $X_{1}$ is smooth, we stop. Otherwise, we let $P_{1}$ be a singular point of $X_{1}$, and $p_{1}=\pi_{1}\left(P_{1}\right)$. Note that $d=2$ or 3 . It implies that $\pi_{1}^{-1}\left(p_{1}\right)=\left\{P_{1}\right\}$ or $\left\{P_{1}, P_{1}^{\prime}\right\}$. In the later case, $d=3, d_{P_{1}}=2, d_{P_{1}^{\prime}}=1$ and $P_{1}^{\prime}$ is a smooth point of $X_{1}$. 
Let $\sigma_{1}: Y_{2} \rightarrow Y_{1}$ be the blowing up of $Y_{1}$ at $p_{1}$, let $X_{2}$ be the normalization of $X_{1} \times_{Y_{1}} Y_{2}$, and let $\bar{\sigma}_{1}$ and $\pi_{2}$ be the induced morphisms. If $X_{2}$ is smooth, we stop. Otherwise, we let $P_{2}$ be a singular point of $X_{2}$ and let $p_{2}=\pi_{2}\left(P_{2}\right)$. We have $\pi_{2}^{-1}\left(p_{2}\right)=\left\{P_{2}\right\}$ or $\left\{P_{2}, P_{2}^{\prime}\right\} . P_{2}^{\prime}$ must be a smooth point of $X_{2}$.

Repeat this process, and after a finite number of steps, $X_{k}$ must be a smooth surface. Therefore, we get a sequence of surface singular points $\left\{P=P_{0}, P_{1}, \cdots, P_{k-1}\right\}$, which are called the infinitely near singular points of $(X, P)$.

We denote by $E=E_{0}, E_{1}, \cdots, E_{k-1}$ the exceptional curves of $\sigma_{0}, \sigma_{1} \cdots, \sigma_{k-1}$, respectively, and by $\mathcal{E}_{i}$ (resp. $\bar{E}_{i}$ ) the total (resp. strict) transformation of $E_{i}$ in $\bar{Y}$. In the canonical resolution, we let $F_{i}$ (resp. $\left.F_{i}^{\prime}\right)$ be the connected components of $\pi^{*}\left(\mathcal{E}_{i}\right)$ corresponding to $P_{i}\left(\right.$ resp. $\left.P_{i}^{\prime}\right)$, i.e.,

$$
\bar{\pi}^{*}\left(\mathcal{E}_{i}\right)= \begin{cases}F_{i}, & \text { if } \pi_{i}^{-1}\left(p_{i}\right)=\left\{P_{i}\right\}, \\ F_{i}+F_{i}^{\prime}, & \text { if } \pi_{i}^{-1}\left(p_{i}\right)=\left\{P_{i}, P_{i}^{\prime}\right\},\end{cases}
$$

where $P_{i}$ is an infinitely near singular point of $(X, P)$, so $d_{P_{i}} \geq 2 . P_{i}^{\prime}$ is always a smooth point of the surface, and $d_{P_{i}^{\prime}}=1$. Let

$$
E=E_{0}, \quad \mathcal{E}=\mathcal{E}_{0}, \quad \bar{E}=\bar{E}_{0}, \quad F=F_{0}, \quad F^{\prime}=F_{0}^{\prime} .
$$

Because $P_{i}^{\prime}$ is a smooth point of $X_{i}$, it is easily to see that $F_{i}^{\prime}$ is the fundamental cycle of the first kind, i.e., a (-1)-cycle,

$$
p_{a}\left(F_{i}^{\prime}\right)=0, \quad{F_{i}^{\prime}}^{2}=-1
$$

$F_{i}$ and $F_{i}^{\prime}$ are disjoint. In fact, we can ignore the smooth points $P_{i}^{\prime}$ and the $(-1)$-cycles $F_{i}^{\prime}$.

In order to compute the maximal ideal cycle, we will decompose $F_{i}$ as a sum of fundamental cycles.

TheOREM 1.1. Let $\pi:(X, P) \rightarrow(Y, p)$ be a local normal finite cover of degree $d=d_{P} \leq 3$ over a smooth surface $Y$ such that $\pi$ is totally ramified over $p=\pi(P)$. Suppose $(X, P)$ is a singularity with multiplicity $m_{P} \geq 2 . \bar{\sigma}: \bar{X} \rightarrow(X, P)$ is the canonical resolution. $\mathcal{E}$ is the total transform of the first exception curve of the blowing ups $\sigma: \bar{Y} \rightarrow Y$. In this case, $F=\bar{\pi}^{*}(\mathcal{E})$ is connected.

Then there are $\ell=\ell_{P} \leq d_{P}$ fundamental cycles $Z=Z_{1}>Z_{2}>\cdots>Z_{\ell}>0$ such that

$$
F=Z_{1}+Z_{2}+\cdots+Z_{\ell}, \quad Z_{i} Z_{j}=0 \text { for } \mathrm{i} \neq \mathrm{j}
$$

Note that $m_{P}<d_{P}$ implies $m_{P}=2$ and $d_{P}=3$.

THEOREM 1.2. With the same notation and assumption as in the previous theorem. Let $M$ be the maximal ideal cycle under the canonical resolution.

1. If $2 \leq m_{P}=d_{P} \leq 3$, then $M=F=Z_{1}+Z_{2}+\cdots+Z_{\ell}$.

2. If $m_{P}=2, d_{P}=3$ and $\ell_{P}=2$, then $M=Z_{1}$.

3. If $m_{P}=2, d_{P}=3$ and $\ell_{P}=3$, then $M=Z_{1}$ or $M=Z_{1}+Z_{2}$.

Definition 1.1. We call $\ell=\ell_{P}$ in the decomposition (2) as the length of $(X, P)$. $Z_{j}$ is called the $j$-th fundamental cycle of $(X, P) . Z_{1}$ is the fundamental cycle in the usual sense. Let

$$
w_{P}:=p_{a}\left(Z_{1}\right)+\cdots+p_{a}\left(Z_{\ell_{P}}\right)+d_{P}-\ell_{P} .
$$


Note that if we set $Z_{\ell_{P}+1}=\cdots=Z_{d_{P}}=0$, then $p_{a}\left(Z_{j}\right)=1$ for $j>\ell_{P}$, and

$$
w_{P}=p_{a}\left(Z_{1}\right)+\cdots+p_{a}\left(Z_{d_{P}}\right) .
$$

In the canonical resolution of $(X, P)$, we get a sequence of infinitely near singular points $\left\{P=P_{0}, P_{1}, \cdots, P_{k-1}\right\}$. Thus we get a sequence of numerical invariants $w=w_{0}, w_{1}, \cdots, w_{k-1}$, where $w_{i}=w_{P_{i}}$.

We would like to point out that $w_{0}, w_{1}, \cdots, w_{k-1}$ are invariants of the singularity $p$ of the branch locus $B_{\pi}$.

If $d=d_{P}=2$, then $w_{i}=\left[m_{i} / 2\right]$ in Horikawa's notation, where $m_{i}^{\prime} s$ are the multiplicities mult $_{p_{i}}\left(B_{\pi_{i}}\right)$ of the singular points of the branch locus. Then we have the well-known formula for the geometric genus of $(X, P)$.

$$
p_{g}(X, P)=\sum_{i=0}^{k-1} \frac{1}{2} w_{i}\left(w_{i}-1\right) .
$$

If $d=d_{P}=3$, then the second author found a similar computation formula for $w_{i}$ by using the singularities of the branch locus, (see $\S 4.3$, or [13], Theorem 6.4.) In fact, (3) or (4) is a unified formula for the invariants $w_{i}$ defined respectively by Horikawa and the second author. It gives a direct relationship between the singularities of the branch locus and the singularities of the surface. Therefore, we can use the invariants $w_{0}, w_{1}, \cdots, w_{k-1}$ of the branch locus to classify the surface singularity $(X, P)$.

TheOREM 1.3. Suppose the surface singularity $(X, P) \rightarrow(Y, p)$ is a finite cover of degree $d=2$ or 3 totally ramified over $p$. Let $P=P_{0}, P_{1}, \cdots, P_{k-1}$ be the infinitely near singular points obtained in the canonical resolution. Then

1. $w_{i} \geq 1$ for $i=0,1, \cdots, k-1$.

2. $(X, P)$ is a rational singular point iff $w_{i} \leq d-1$ for any $i$.

3. If $(X, P)$ is rational, then the multiplicity of $(X, P)$ is $w_{0}+1$.

In order to get the minimal resolution $\eta: S \rightarrow(X, P)$, we need to blow down the $(-1)$-curves in the exceptional set of the canonical resolution, $\bar{\tau}: \bar{X} \rightarrow S$.

The first step is to contract the obvious $(-1)$-cycles $F_{i}^{\prime}$. We get a smooth surface $\tilde{X}, \tau^{\prime}: \bar{X} \rightarrow \widetilde{X}$. Then we need to contract the $(-1)$-curves in $F_{0}, F_{1}, \cdots, F_{k-1}$, $\tau: \widetilde{X} \rightarrow S$, we get the minimal resolution $\eta: S \rightarrow(X, P)$.

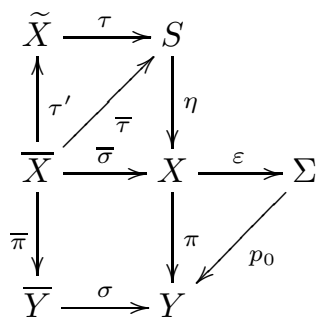

Definition 1.2. $J=\eta \circ \tau: \widetilde{X} \rightarrow X$ is usually called the Jung's resolution of $(X, P)$.

Because $\tau$ consists of blowing ups at smooth points, the total transformations $\mathcal{D}_{1}, \cdots, \mathcal{D}_{r}$ of the exceptional curves $D_{1}, \cdots, D_{r}$ of $\tau$ are $(-1)$-cycles in $\widetilde{X}$. The number $r=r(J)$ of curves contracted by $\tau$ is determined by the Jung's resolution $J$. 
The following fundamental problems remain open (see [10]).

Problem 1.1. Adapt Jung's resolution to get embedded resolution of germs of surfaces $\Sigma$ in $\mathbb{C}^{3}$

Problem 1.2. Use Jung's resolution to get obstructions on the topology of local surfaces with isolated singularities in $\mathbb{C}^{3}$.

Problem 1.3. Fix the topology of $(X, P)$. Is $r=r(J)$ bounded from above? Compute it in terms of the weighted dual graph of the minimal good resolution of $(X, P)$ (which encodes the topology of $(X, P)$, as ensured by a theorem of Neumann).

Suppose the decompositions of $F_{0}, F_{1}, \cdots, F_{k-1}$ are as follows.

$$
\begin{gathered}
F_{0}=Z_{1}+\cdots+Z_{\ell_{0}}, \\
F_{1}=Z_{1}^{\prime}+\cdots+Z_{\ell_{1}}^{\prime}, \\
F_{2}=Z_{1}^{\prime \prime}+\cdots+Z_{\ell_{2}}^{\prime \prime}, \\
\vdots \\
F_{k-1}=Z_{1}^{(k-1)}+\cdots+Z_{\ell_{k-1}}^{(k-1)} .
\end{gathered}
$$

We will prove that all of the components $Z_{j}^{(i)}$ are different. Let

$$
\mathcal{F u n d}(J):=\left\{Z_{j}^{(i)} \mid \text { for any } i, j\right\}
$$

TheOREM 1.4. $\mathcal{D}_{1}, \cdots, \mathcal{D}_{r}$ are exactly the $(-1)$-cycles in the set $\mathcal{F} u n d(J)$ of fundamental cycles.

$$
\left\{\mathcal{D}_{1}, \cdots, \mathcal{D}_{r}\right\}=\left\{Z \in \mathcal{F} \text { und }(J) \mid p_{a}(Z)=0, Z^{2}=-1\right\} .
$$

So the number $r=r(J)$ of curves contracted by $\tau$ can be computed from the decompositions. This result allows us to determine the curves contracted by $\tau$ from the singularities of the branch locus. For a surface singularity of multiplicity 2, Xiao determined the number $r$ of the curves contracted by $\tau$ using a different method. Xiao proves that $r$ is equal to the number of singularities of types $(2 w+1 \rightarrow 2 w+1)$ of the branch locus. In fact, such a singular point has positive contribution to the geometric genus. As a consequence, we get

$$
r(J) \leq p_{g}(X, P) .
$$

We will try to get a similar classification for surface singularities of multiplicity 3 .

\section{Fundamental cycles and the canonical resolution.}

2.1. Fundamental cycles. Let $(X, P)$ be an isolated surface singularity and $\bar{\sigma}:\left(\bar{X}, E_{P}\right) \rightarrow(X, P)$ be a resolution, where $E_{P}$ is the set of exceptional curves. There is a unique divisor $Z$ supported on $E_{P}$ such that $Z \Gamma \leq 0$ for any component $\Gamma$ in $E_{P}$, and $Z$ is minimal with respect to this property (see [2]) or [7, Sec.4.5]). Such a minimal cycle $Z$ is called the fundamental cycle of $E_{P}$, or of the resolution. 
In general, for a connected subset $E^{\prime}$ of $E_{P}$, we can also define a fundamental cycle $Z^{\prime}$ whose support is $E^{\prime}$, i.e., $Z^{\prime}$ is a minimal cycle satisfying $Z^{\prime} \Gamma \leq 0$ for any curve $\Gamma$ in $E^{\prime}$. We simply say that $Z^{\prime}$ is a fundamental cycle.

We can obtain $Z$ by the computational sequence: Let $Z_{1}$ be any fundamental cycle of its support (e.g., $Z_{1}$ is one component of the exceptional set), choose a component $\Gamma_{1}$ such that $Z_{1} \Gamma_{1}>0$, and let $Z_{2}=Z_{1}+\Gamma_{1}$. Choose a component $\Gamma_{2}$ such that $Z_{2} \Gamma_{2}>0$ and let $Z_{3}=Z_{2}+\Gamma_{2}, \cdots$, after a finite number of steps, we get $Z=Z_{\ell}$. It is well-known that $p_{a}\left(Z_{i}\right)=h^{1}\left(\mathcal{O}_{Z_{i}}\right)$ and

$$
p_{a}(Z)=p_{a}\left(Z_{\ell}\right) \geq p_{a}\left(Z_{\ell-1}\right) \geq \cdots \geq p_{a}\left(Z_{1}\right) \geq 0
$$

Lemma 2.1. If $Z^{\prime}$ and $Z$ are two fundamental cycles, and the support of $Z^{\prime}$ is contained in that of $Z$, then $Z^{\prime} \leq Z$ and $p_{a}\left(Z^{\prime}\right) \leq p_{a}(Z)$.

Lemma 2.2. Let $A$ and $B$ be positive cycles supported on $E_{P}$. Suppose $A$ is connected and $B C \leq 0$ for any component $C$ in $A$. Then either the support of $A$ is contained in that of $B$, or $A$ and $B$ are disjoint.

Proof. Let $A=A_{1}+A_{2}$ be the decomposition such that the support of $A_{1}$ is contained in $B$, and $A_{2}$ has no common component with $B$. Then we have $A_{2} B \geq 0$. By assumption, $A_{2} B \leq 0$, so $A_{2} B=0$ and $A_{2}$ are disjoint from $B$. Hence $A_{2}$ is disjoint from $A_{1}$. Because $A$ is connected, we get either $A_{1}=0$ or $A_{2}=0$. This is what we desired.

Lemma 2.3. Suppose $A$ and $B$ are two distinct positive cycles supported on $E_{P}$. If $A^{2}=B^{2}=-1$, then $A B=0$. Furthermore, if $A$ and $B$ are not disjoint, then $A \geq B$ or $B \geq A$.

Proof. Because $A \neq B, A \pm B$ is not zero, we have

$$
(A \pm B)^{2}=-1-1 \pm 2 A B<0,
$$

so $A B=0$.

If $A$ and $B$ are not disjoint, then they have at least one common component. Let $C$ be the biggest common positive cycle such that $A \geq C$ and $B \geq C$. Let $A^{\prime}=A-C$ and $B^{\prime}=B-C$. Then $A^{\prime}$ and $B^{\prime}$ have no common component. So $A^{\prime} B^{\prime} \geq 0$. Now we claim that either $A^{\prime}=0$ or $B^{\prime}=0$. Otherwise, $A^{\prime 2} \leq-1$ and $B^{\prime 2} \leq-1$.

$$
-2=(A-B)^{2}=\left(A^{\prime}-B^{\prime}\right)^{2}=A^{\prime 2}+B^{\prime 2}-2 A^{\prime} B^{\prime} \leq-2,
$$

so $A^{\prime} B^{\prime}=0, A^{\prime 2}=-1$ and $B^{\prime 2}=-1$. Note that $A^{\prime} \neq B, B^{\prime} \neq A$, and $A^{2}=B^{2}=$ $A^{\prime 2}=B^{\prime 2}=-1$, by the proof of the first part, we get $A B^{\prime}=A^{\prime} B=0$. So

$$
C^{2}=\left(A-A^{\prime}\right)\left(B-B^{\prime}\right)=A B+A^{\prime} B^{\prime}-A B^{\prime}-A B^{\prime}=0,
$$

it implies $C=0$, a contradiction.

2.2. Maximal ideal cycle, canonical cycle and $(-n)$-cycles. The maximal ideal cycle $M$ of $E_{P}$ is defined as the greatest divisor contained in every divisor of type $\operatorname{div}\left(\bar{\sigma}^{*} g\right)$, where $g$ is any nonzero holomorphic function on $X$ with $g(P)=0$. Namely,

$$
M=\operatorname{gcd}\left\{\operatorname{div}\left(\bar{\sigma}^{*} g\right) \mid 0 \neq g \in m_{P} \subseteq \mathcal{O}_{X, P}\right\} .
$$


It is easy to see that $Z \leq \operatorname{div}\left(\bar{\sigma}^{*} g\right)$ for any $g$ in $m_{P}$, so $Z \leq M$. The equality holds in some important cases: for example, rational singularities and elliptic Gorenstein singularities (see [7, Theorem 4.17 and 4.23]). If $m_{P} \mathcal{O}_{Y}=\mathcal{O}_{Y}(-M)$, then $\operatorname{mult}_{P}(X)=-M^{2}$ (see [7, Page 85]).

Let $K$ be the unique $\mathbb{Q}$-divisor supported on the exceptional set $E_{P}$ such that $K \Gamma+\Gamma^{2}=2 p_{a}(\Gamma)-2$ for any component $\Gamma$ of $E_{P} . K$ is called the canonical cycle of $(X, P)$.

An effective divisor $D$ supported on some exceptional curves in $E_{P}$ is called a $(-n)$-cycle if $D$ is the fundamental cycle of its support and

$$
p_{a}(D)=0, \quad D^{2}=-n .
$$

By Artin's theorem [2], (-1)-cycle can be contracted to a smooth point of a surface. It is well-known that the arithmetic genus $p_{a}(Z) \geq 0$ for any fundamental cycle $Z$.

2.3. Canonical resolution of double and triple covers. In what follows, we will try to compute the cycles defined in the previous section for the canonical resolution $\bar{\sigma}: \bar{X} \rightarrow(X, P)$ explained in the introduction.

Note that in the Picard group of $\bar{Y},\left\{\bar{E}_{0}, \cdots, \bar{E}_{k-1}\right\}$ and $\left\{\mathcal{E}_{0}, \cdots, \mathcal{E}_{k-1}\right\}$ generate the same subgroup.

$$
\mathbb{Z} \bar{E}_{0}+\cdots+\mathbb{Z} \bar{E}_{k-1}=\mathbb{Z} \mathcal{E}_{0}+\cdots+\mathbb{Z} \mathcal{E}_{k-1}
$$

As generators, $\mathcal{E}_{0}, \cdots, \mathcal{E}_{k-1}$ are convenient for computation. For any $i$ and $j \neq i$, we have

$$
\mathcal{E}_{i}^{2}=-1, \quad \mathcal{E}_{i} \cdot \mathcal{E}_{j}=0 .
$$

$\mathcal{E}_{i}$ is a $(-1)$-fundamental cycle. For $i>j$, either $\mathcal{E}_{i}$ and $\mathcal{E}_{j}$ are disjoint, or $\mathcal{E}_{i}<\mathcal{E}_{j}$. For any exceptional curve $\Gamma$ of $\sigma$ in $\bar{Y}$,

$$
\Gamma \cdot \mathcal{E}_{i}= \begin{cases}-1, & \text { if } \Gamma=\bar{E}_{i}, \\ 0, & \text { if } \Gamma \neq \bar{E}_{i} \text { is contained in } \mathcal{E}_{i} \\ \geq 0, & \text { otherwise. }\end{cases}
$$

LemmA 2.4. In the subgroup $\mathbb{Z} \bar{E}_{0}+\cdots+\mathbb{Z} \bar{E}_{k-1}$ of $\operatorname{Pic}(\overline{\mathrm{Y}}), \mathcal{E}_{0}, \cdots, \mathcal{E}_{k-1}$ are the only effective divisors whose self-intersection numbers are -1 .

Proof. Let $D$ be an effective divisor in this subgroup, by (5), we can write $D=$ $n_{0} \mathcal{E}_{0}+\cdots+n_{k-1} \mathcal{E}_{k-1}$ for some integers $n_{0}, \cdots, n_{k-1}$. If $D^{2}=-1$, then we have

$$
-1=-n_{0}^{2}-\cdots-n_{k-1}^{2},
$$

so there exists an $i$ such that $n_{i}= \pm 1, n_{j}=0$ for $j \neq i$. Because $D$ is effective, $D=\mathcal{E}_{i}$.

LEMma 2.5. Let $Z$ be a fundamental cycle containing the support of a (-1)-cycle $\mathcal{D}$ on a smooth surface $X$, and let $\gamma: X \rightarrow S$ be the contraction map of the curves in $\mathcal{D}$ to a smooth surface $S$. Then either $Z=\mathcal{D}$ or $Z=\gamma^{*} \gamma_{*}(Z)$. In particular, if $Z \neq \mathcal{D}$, then $Z \mathcal{D}=0$. 
Proof. Suppose $Z \neq \mathcal{D}$. From the uniqueness of the fundamental cycle, we see that $\operatorname{Supp}(\mathcal{D}) \varsubsetneqq \operatorname{Supp}(Z)$. We see that at least one curve $C$ in $Z$ is not contracted by $\gamma$. For such a curve $C$,

$$
C \cdot \gamma^{*} \gamma_{*}(Z)=\gamma^{*} \gamma_{*}(C) \cdot \gamma^{*} \gamma_{*}(Z)=\gamma^{*} \gamma_{*}(C) \cdot Z \leq 0 .
$$

If $C^{\prime}$ is contracted by $\gamma$, then $C^{\prime} \cdot \gamma^{*} \gamma_{*}(Z)=0$. From the minimality of fundamental cycle, we have that $Z \leq \gamma^{*} \gamma_{*}(Z)$, so $\gamma^{*} \gamma_{*}(Z)=Z+A$ for some effective divisor $A$ whose support consists of curves contracted by $\gamma$. Hence

$$
0=\gamma^{*} \gamma_{*}(Z) A=Z A+A^{2} \leq A^{2}
$$

we have $A^{2}=0$ and $A=0$. Thus $Z=\gamma^{*} \gamma_{*}(Z)$ and $Z \mathcal{D}=0$.

2.4. Computation of the maximal ideal cycle. The following theorem is known for surface singularities of multiplicity 2 .

Theorem 2.1. Let $(X, P)$ be a normal surface singularity, let $\pi:(X, P) \rightarrow(Y, p)$ be a finite cover of degree $d=d_{P} \leq 3$ over a smooth surface $Y$ totally ramified over $p=\pi(P)$, and let $\bar{\sigma}: \bar{X} \rightarrow X$ be the canonical resolution. Let $F=\bar{\pi}^{*} \mathcal{E}=\bar{\pi}^{*} \mathcal{E}_{0}$. If the multiplicity $m_{P}$ of $(X, P)$ is equal to the local degree $d_{P}$, then the maximal ideal cycle $M$ of $(X, P)$ under $\bar{\sigma}$ is equal to $F$.

Proof. Because the case when $d=2$ is well-known, we assume that $d=3$. By [9], $X$ is a local surface in $\mathbb{C}^{4}$ defined by the following equations.

$$
\left\{\begin{array}{l}
z^{2}=a z+b w+2 A, \\
z w=-d z-a w-B, \\
w^{2}=c z+d w+2 C,
\end{array}\right.
$$

where $a, b, c, d \in \mathcal{O}_{Y, p}$ and $A=a^{2}-b d, B=a d-b c$ and $C=d^{2}-a c$. Suppose $m_{p} \subset \mathcal{O}_{Y, p}$ is generated by $x$ and $y$, then $m_{P} \subset \mathcal{O}_{X, P}$ is generated by $x, y, z$ and $w$. Since $\pi$ is totally ramified over $p$, we have $A(p)=B(p)=C(p)=0$.

If $b(p) \neq 0$, then $b$ is invertible, from the defining equations, we can eliminate $w$. Namely, $(X, P)$ is a hypersurface singularity defined by

$$
z^{3}+s z+t=0
$$

where $s=-3 A$ and $t=b B-2 a A$. Because $\operatorname{mult}_{P}(X)=3$, we have $\nu_{p}(s) \geq 2$ and $\nu_{p}(t) \geq 3$. Now from

$$
\bar{\sigma}^{*}(z)^{3}+\bar{\pi}^{*}\left(\sigma^{*}(s)\right) \bar{\sigma}^{*}(z)+\bar{\pi}^{*}\left(\sigma^{*}(t)\right)=0,
$$

we see that for any element $g \in m_{p}$ with $\nu_{p}(g)=1$, we have

$$
\nu_{\Gamma}\left(\bar{\sigma}^{*}(z)\right) \geq \nu_{\Gamma}\left(\bar{\pi}^{*} \sigma^{*}(g)\right)
$$

for any exceptional curve $\Gamma$ of $\bar{\sigma}$. In particular,

$$
\nu_{\Gamma}\left(\bar{\sigma}^{*}(z)\right) \geq \nu_{\Gamma}\left(\bar{\pi}^{*} \sigma^{*}(x)\right), \quad \nu_{\Gamma}\left(\bar{\sigma}^{*}(z)\right) \geq \nu_{\Gamma}\left(\bar{\pi}^{*} \sigma^{*}(y)\right) .
$$

In this case, $m_{P}$ is generated by $x, y$ and $z$. Hence

$$
\begin{aligned}
F & =\operatorname{gcd}\left\{\operatorname{div}\left(\bar{\sigma}^{*}\left(\pi^{*}(x)\right)\right), \operatorname{div}\left(\bar{\sigma}^{*} \pi^{*}(y)\right), \operatorname{div}\left(\bar{\sigma}^{*}(z)\right)\right\} \\
& =\operatorname{gcd}\left\{\operatorname{div}\left(\bar{\pi}^{*}\left(\sigma^{*}(x)\right)\right), \operatorname{div}\left(\bar{\pi}^{*} \sigma^{*}(y)\right), \operatorname{div}\left(\bar{\sigma}^{*}(z)\right)\right\} \\
& =\operatorname{gcd}\left\{\operatorname{div}\left(\bar{\pi}^{*}\left(\sigma^{*}(x)\right)\right), \operatorname{div}\left(\bar{\pi}^{*} \sigma^{*}(y)\right)\right\} \\
& =\bar{\pi}^{*}\left(\mathcal{E}_{0}\right) .
\end{aligned}
$$


If $c(p) \neq 0$, the proof is similar.

Now suppose $b(p)=c(p)=0$, from $A(p)=B(p)=C(p)=0$, we have also $a(p)=0$ and $d(p)=0$. Now from the defining equation (8), we can get (10) and

$$
\nu_{\Gamma}\left(\bar{\sigma}^{*}(w)\right) \geq \nu_{\Gamma}\left(\bar{\pi}^{*} \sigma^{*}(x)\right), \quad \nu_{\Gamma}\left(\bar{\sigma}^{*}(w)\right) \geq \nu_{\Gamma}\left(\bar{\pi}^{*} \sigma^{*}(y)\right) .
$$

We obtain similarly that $F=\bar{\pi}^{*}\left(\mathcal{E}_{0}\right)$.

2.5. Decomposition of $F$. Let $\pi:(X, P) \rightarrow(Y, p)$ be a normal finite cover of degree $d \leq 3$ over a smooth surface $Y$ such that $\pi$ is totally ramified over $p=\pi(P)$, let $\bar{\sigma}: \bar{X} \rightarrow X$ be the canonical resolution.

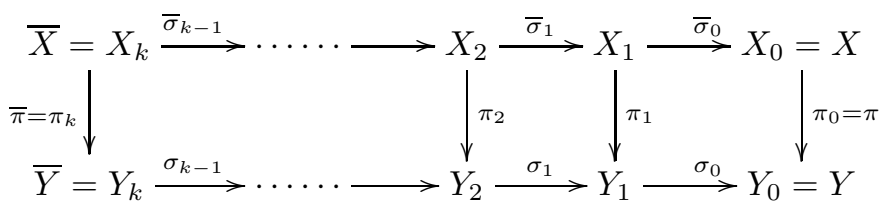

We have defined the local degree $d=d_{P}$, the length $\ell=\ell_{P}$ and the multiplicity $m_{P}$ of $(X, P) . F_{P}=\pi^{*} \mathcal{E}_{P}$, and $M_{P}$ is the maximal ideal cycle of $(X, P)$ under the canonical resolution $\bar{\sigma}$.

Note that we have a sequence of infinitely near singular points $P=$ $P_{0}, P_{1}, \cdots, P_{k-1}$ with local degrees $d=d_{0}, d_{1}, \cdots, d_{k-1}$, local lengths $\ell=$ $\ell_{0}, \ell_{1}, \cdots, \ell_{k-1}$ and multiplicities $m=m_{0}, m_{1}, \cdots, m_{k-1}$. The corresponding sequence of connected cycles are denoted by $F=F_{0}, F_{1}, \cdots, F_{k-1}$.

TheOREM 2.2. Suppose $d=d_{0} \leq 3$. Then there are $\ell=\ell_{0} \leq d$ fundamental cycles $Z_{1}>Z_{2}>\cdots>Z_{\ell_{0}}>0$ such that

$$
F=F_{0}=Z_{1}+Z_{2}+\cdots+Z_{\ell_{0}}, \quad Z_{i} Z_{j}=0 \text { for } \mathrm{i} \neq \mathrm{j} .
$$

The decomposition is unique. In particular, we have

$$
p_{a}\left(Z_{1}\right) \geq p_{a}\left(Z_{2}\right) \geq \cdots \geq p_{a}\left(Z_{\ell_{0}}\right) \geq 0 .
$$

Proof. We have proved that $F_{0}=\bar{\pi}^{*}\left(\mathcal{E}_{0}\right)$. So $F_{0}^{2}=-d_{0} \geq-3$. Note that for any component $\Gamma$ of $F_{0}$, we have $\Gamma F_{0} \leq 0$. The fundamental cycle $Z_{1}=Z$ supported on $F_{0}$ is the minimal effective divisor satisfying $Z \Gamma \leq 0$. So $Z_{1} \leq F_{0}$ and $A:=F_{0}-Z_{1}$ is an effective divisor, which implies that $A Z_{1} \leq 0$.

$$
-3 \leq F_{0}^{2}=Z_{1}^{2}+2 Z_{1} A+A^{2} \leq Z_{1}^{2} .
$$

If $A=0$, the proof is completed. We assume that $A \neq 0$. Thus $A^{2}<0$. Since $F_{0}^{2} \geq-3$, we have

$$
-3 \leq A^{2}+Z_{1}^{2}+2 A Z_{1} \leq-2+2 A Z_{1},
$$

so $A Z_{1} \geq 0$. Because $A Z_{1} \leq 0$, we have $A Z_{1}=0$, which implies $\Gamma Z_{1}=0$ for each component $\Gamma \leq A$. Thus

$$
\Gamma \cdot A=\Gamma \cdot F_{0}-\Gamma \cdot Z_{1}=F_{0} \cdot \Gamma=\mathcal{E}_{1} \cdot \bar{\pi}_{*} \Gamma \leq 0 .
$$

From $F_{0}^{2}=Z_{1}^{2}+A^{2}$, we get $A^{2}=-1$ or -2 . 
Let $Z_{2}$ be the fundamental cycle on the support of $A$. Then $Z_{2} \leq A$, so $Z_{2} Z_{1}=0$. Let $B=A-Z_{2}$. If $B=0$, we are done. Suppose $B \neq 0$. Then $B^{2} \leq-1$ and $B Z_{2} \leq 0$. From

$$
-2 \leq A^{2}=Z_{2}^{2}+2 B Z_{2}+B^{2} \leq-2+2 B Z_{2} \leq-2,
$$

we see that $B Z_{2}=0, B^{2}=Z_{2}^{2}=-1, A^{2}=-2$. Since $B Z_{2}=0$, we get $\Gamma Z_{2}=0$ for any component $\Gamma \leq B$, and

$$
\Gamma B=\Gamma A-\Gamma Z_{2}=\Gamma A \leq 0 .
$$

Let $Z_{3}$ be the fundamental cycle of the support of $B$. Then we have $Z_{3} Z_{2}=$ $Z_{3} Z_{1}=0$. Take $C=B-Z_{3}$. Then $C Z_{3} \leq 0$.

$$
-1=B^{2}=Z_{3}^{2}+2 C Z_{3}+C^{2} \leq-1+C^{2} \leq-1,
$$

so $C^{2}=0$, which implies $C=0$.

Therefore, $Z_{i} Z_{j}=0(i \neq j)$. By Lemma $2.1, Z_{i} \geq Z_{i+1}$. Because $Z_{i} \cdot Z_{i+1}=0$, we have $Z_{i}>Z_{i+1}$. Hence

$$
d_{0}=-F_{0}^{2}=-Z_{1}^{2}-Z_{2}^{2}-\cdots-Z_{\ell_{0}}^{2} \geq \ell_{0}
$$

The inequalities (13) are well-known facts about fundamental cycles (see Lemma 2.1).

Note that $-F_{P}$ is nef with respect to the exceptional curves. From the theorem, $Z_{i}^{2}=Z_{i} F$, thus we have

$$
Z_{1}^{2} \leq Z_{2}^{2} \leq \cdots \leq Z_{\ell}^{2}
$$

Since $F^{2}=Z_{1}^{2}+\cdots+Z_{\ell}^{2}=-d$, we see that if $Z_{1}^{2}=-1$, then $\ell=d$ and $Z_{i}^{2}=-1$ for any $i$.

THEOREM 2.3. With the notations as in the previous theorem, we have

1. If $d_{P}=3, m_{P}=2$ and $\ell_{P}=2$, then $M_{P}=Z_{1}$.

2. If $d_{P}=3, m_{P}=2$ and $\ell_{P}=3$, then $M_{P}=Z_{1}$ or $M_{P}=Z_{1}+Z_{2}$.

Proof. We use the well-known fact that $-M_{P}^{2} \leq m_{P}=2$. Since $F_{P}{ }^{2}=-3$, we see that $M_{P} \neq F_{P}$. Note that $M_{P} \Gamma \leq 0$ for any component in the exceptional set. So $M_{P} \geq Z_{1}$. If $M_{P}=Z_{1}$, then (1) and (2) are proved.

Now we assume that $M_{P}=Z_{1}+D$ for some effective nonzero divisor $D$.

$$
-2 \leq M_{P}^{2}=Z_{1}^{2}+D^{2}+2 Z_{1} D \leq-1-1,
$$

we have $M_{P}^{2}=-2, Z_{1}^{2}=D^{2}=-1$ and $Z_{1} D=0$. So $\ell_{P}=3, F_{P}=Z_{1}+Z_{2}+Z_{3}$, and $Z_{1}^{2}=Z_{2}^{2}=Z_{3}^{2}=-1$.

Now we prove that $D \neq Z_{3}$. Otherwise, $M_{P}=Z_{1}+Z_{3},\left(Z_{2}-Z_{3}\right) M_{P}=-Z_{3}^{2}=1$, which contradicts the nefness of $-M_{P}$ on the exceptional set. By Lemma 2.3, we get that $D Z_{3}=0$.

Suppose $D \neq Z_{2}$, by Lemma 2.3, we have $D Z_{2}=0$. Then $D F_{P}=D Z_{1}+D Z_{2}+$ $D Z_{3}=0$. So $F_{P} M_{P}=F_{P} Z_{1}=-1$.

On the other hand, take a generic smooth curve $C$ on $Y$ passing through $p$, and we let $g=0$ is its defining equation. Then $\pi^{*}(g)$ is a holomorphic function on $X$ vanishing on $P$. One can see that $\operatorname{div}\left(\bar{\sigma}^{*}\left(\pi^{*}(g)\right)=\operatorname{div}\left(\bar{\pi}^{*}\left(\sigma^{*}(g)\right)\right)=F+\bar{C}\right.$, where 
$\bar{C}$ is the strict transform of the curve $C$ on $\bar{X}$. Thus $F_{P} \geq M_{P}$, which implies that $1=-1-(-2)=\left(F_{P}-M_{P}\right) M_{P} \leq 0$, a contradiction.

Therefore, $D=Z_{2}$ and $M_{P}=Z_{1}+Z_{2}$. This completes the proof.

Suppose the decompositions of $F_{0}, F_{1}, \cdots$ are as follows.

$$
\begin{gathered}
F_{0}=Z_{1}+\cdots+Z_{\ell_{0}} \\
F_{1}=Z_{1}^{\prime}+\cdots+Z_{\ell_{1}}^{\prime} \\
F_{2}=Z_{1}^{\prime \prime}+\cdots+Z_{\ell_{2}}^{\prime \prime} \\
\vdots \\
F_{k-1}=Z_{1}^{(k-1)}+\cdots+Z_{\ell_{k-1}}^{(k-1)}
\end{gathered}
$$

Corollary 2.1. $Z_{j}^{(i)}=Z_{j^{\prime}}^{\left(i^{\prime}\right)}$ if and only if $i=i^{\prime}$ and $j=j^{\prime}$.

Proof. Suppose that $Z_{j}^{(i)}=Z_{j^{\prime}}^{\left(i^{\prime}\right)}>0$. Let $\bar{\pi}^{*} \mathcal{E}_{i}=Z_{j}^{(i)}+A, \bar{\pi}^{*} \mathcal{E}_{i^{\prime}}=Z_{j}^{(i)}+B$. From Theorem 2.2, if $i=i^{\prime}$, then $j=j^{\prime}$. Now we assume that $i^{\prime} \neq i$, so $\mathcal{E}_{i^{\prime}} \cdot \mathcal{E}_{i}=0$. Then $A-B=\bar{\pi}^{*} \mathcal{E}_{i}-\bar{\pi}^{*} \mathcal{E}_{i^{\prime}}$ implies

$$
(A-B)^{2}=\left(\bar{\pi}^{*} \mathcal{E}_{i}\right)^{2}+\left(\bar{\pi}^{*} \mathcal{E}_{i^{\prime}}\right)^{2}=-2 d_{0} \leq-4 .
$$

If $Z_{j}^{(i)} \cdot Z_{j}^{(i)}=-d_{0}$, then $A=B=0$, and $(A-B)^{2}=0$, a contradiction. If $Z_{j}^{(i)} \cdot Z_{j}^{(i)}=-d_{0}+1$, then $A^{2}=B^{2}=-1$, hence $A B=0$ by Lemma 2.3. So $(A-B)^{2}=-2$, a contradiction. Therefore $Z_{j}^{(i)} \cdot Z_{j}^{(i)} \geq-d_{0}+2$. It implies $d_{0}=3$ and $Z_{j}^{(i)} \cdot Z_{j}^{(i)}=-1$. From Theorem $2.2, A^{2}=B^{2}=-2$ and $Z_{j^{\prime}}^{\left(i^{\prime}\right)} \cdot B=Z_{j}^{(i)} \cdot B=0$.

Without a loss of generality, we assume that $\mathcal{E}_{i}>\mathcal{E}_{i^{\prime}}$. So $\bar{\pi}^{*} \mathcal{E}_{i} \cdot B=\mathcal{E}_{i} \cdot \bar{\pi}_{*} B=0$. Thus $A B=\bar{\pi}^{*} \mathcal{E}_{i} \cdot B-Z_{j}^{(i)} \cdot B=0$. On the other hand, $(A-B)^{2}=-6$ implies $A B=1$, a contradiction.

We call $\left(d_{P}, \ell_{P}\right)$ the type of the singularity $(X, P)$ or the local finite cover $(X, P) \rightarrow$ $(Y, p)$. For the infinitely near singular points $P=P_{0}, P_{1}, \cdots, P_{k-1}$ in the canonical resolution, we have a sequence of types:

$$
\left(d_{0}, \ell_{0}\right),\left(d_{1}, \ell_{1}\right), \cdots,\left(d_{k-1}, \ell_{k-1}\right) .
$$

In what follows, we are trying to find the relationships between the types and fundamental cycles of $(X, P)$ and $\left(X_{1}, P_{1}\right)$.

\subsection{The case $\ell_{0}=d_{0}$.}

Corollary 2.2. Let $\Gamma$ be an irreducible component of $Z_{\ell_{0}}$ such that $Z_{\ell_{0}} \Gamma<0$. Then we have $\bar{\pi}^{*} \bar{E}_{0} \geq \ell_{0} \Gamma$. Therefore, if $\ell_{0}=\ell_{P_{0}} \geq 2$, then $\bar{E}_{0}$ lies in the branch locus.

Moreover, if $\ell_{P_{0}}=d_{0}$, then $\bar{\pi}$ is totally ramified over $\bar{E}_{0}$ and the multiplicity of $\Gamma$ in each $Z_{i}$ is 1 . In particular, $\bar{\pi}^{*} \bar{E}_{0}=d_{0} \Gamma$.

Proof. Since $Z_{\ell} Z_{i}=0$ and $Z_{\ell_{0}}<Z_{i}$ for any $i<\ell_{0}, \Gamma Z_{i}=0$. Thus

$$
\mathcal{E}_{0} \cdot \bar{\pi}_{*} \Gamma=\bar{\pi}^{*} \mathcal{E}_{0} \cdot \Gamma=Z_{\ell_{0}} \Gamma<0 .
$$

So $\bar{\pi}(\Gamma)=\bar{E}_{0}$. Since $\Gamma \leq Z_{i}$ for each $i, \bar{\pi}^{*} \mathcal{E}_{0}=Z_{1}+\cdots+Z_{\ell_{0}} \geq \ell_{0} \Gamma$. Hence $\bar{\pi}^{*} \bar{E}_{0} \geq \ell_{0} \Gamma$, i.e., $\pi_{1}$ is ramified over $E_{0}$. Furthermore, if $\ell_{0}=d_{0}$, then $\bar{\pi}^{*} \bar{E}_{0}=d_{0} \Gamma$, 
i.e., $\pi_{1}$ is totally ramified over $E_{0}$. In particular, the multiplicity of $\Gamma$ in each $Z_{i}$ is 1.

Theorem 2.4. Suppose $\ell_{0}=d_{0} \leq 3$. Then each fundamental cycle $Z_{i}$ in $F_{0}=$ $Z_{1}+\cdots+Z_{\ell_{0}}$ satisfies $Z_{i}^{2}=-1$ and $\pi_{1}$ is totally ramified over $E_{0}$. Suppose $P_{1}$ is any singular point of $X_{1}$. Then $\pi_{1}^{-1}\left(p_{1}\right)=\left\{P_{1}\right\}$ and $\ell_{1}=\ell_{P_{1}} \geq d_{0}-1$.

1. If $\ell_{1}=d_{0}-1$, then $Z_{1}^{\prime}=Z_{1}-Z_{\ell_{0}}$. Therefore we have

$$
Z_{1}^{\prime 2}=-2, \quad Z_{1}^{\prime} Z_{1}=-1, \quad Z_{1}^{\prime} Z_{\ell_{0}}=1 .
$$

2. If $\ell_{1}=d_{0}$, then $Z_{i}^{\prime} Z_{j}=0$ for any $i$ and $j$, and

$$
Z_{\ell_{1}}^{\prime}<Z_{\ell_{1}-1}^{\prime}<\cdots<Z_{1}^{\prime}<Z_{\ell_{0}}<Z_{\ell_{0}-1}<\cdots<Z_{2}<Z_{1} .
$$

3. $X_{1}$ admits a singular point, say $P_{1}$, of type (1).

Proof. Since $-Z_{i}^{2} \geq 1\left(i=1, \cdots, \ell_{0}=d_{0}\right)$ and

$$
d_{0}=-Z_{1}^{2}-Z_{2}^{2}-\cdots-Z_{d_{0}}^{2}
$$

we have $-Z_{i}^{2}=1$ for all $i$.

Let $D=Z_{1}-Z_{\ell_{0}}$. Let $\Gamma$ be a component of $Z_{\ell_{0}}$ with $Z_{\ell_{0}} \Gamma<0$. By Corollary 2.2, $D$ does not contain $\Gamma$. Since $Z_{\ell_{0}}^{2}=-1, Z_{\ell_{0}}$ is connected and $\Gamma$ is the only component with $\Gamma \cdot Z_{\ell_{0}}<0$. For any component $C \neq \Gamma$ of $Z_{1}, Z_{\ell_{0}} C \geq 0$ and $Z_{1} C \leq 0$. We see that $D C \leq 0$. Applying Lemma 2.2 to $A=Z_{1}^{\prime}$ and $B=D$, we see that either $D \geq Z_{1}^{\prime}$ or $D$ and $Z_{1}^{\prime}$ are disjoint.

We claim that $C Z_{1}^{\prime} \leq 0$ for any irreducible exceptional component $C \neq \Gamma$. Otherwise, $C Z_{1}^{\prime}>0$ implies $Z_{1}^{\prime}$ doesn't contain $C$ and hence $\bar{\pi}_{*} C \cdot \mathcal{E}_{1}=C \cdot \bar{\pi}^{*} \mathcal{E}_{1}>0$. Thus $\bar{\pi}(C)=\bar{E}_{0}$ by (7), which implies $C=\Gamma$ by Corollary 2.2, a contradiction.

(1) Assume that $D \geq Z_{1}^{\prime}$. Let $D^{\prime}=D-Z_{1}^{\prime}$. Since $D^{\prime}$ does not contain $\Gamma$, the support of $D^{\prime}$ is contained in $Z_{1}^{\prime}$. Thus $D^{\prime} Z_{1}^{\prime} \leq 0$, and

$$
-2=D^{2}=D^{\prime 2}+Z_{1}^{\prime 2}+2 D^{\prime} Z_{1}^{\prime}
$$

If $D^{\prime} Z_{1}^{\prime}<0$, then $D^{\prime 2}=Z_{1}^{\prime 2}=0$, a contradiction. So $D^{\prime} Z_{1}^{\prime}=0$.

Now we claim that $D^{\prime}=0$, i.e., $D=Z_{1}^{\prime}$. Otherwise, $D^{\prime 2}=Z_{1}^{\prime 2}=-1$. Hence $Z_{1}^{\prime} D=Z_{1}^{\prime} D^{\prime}+Z_{1}^{\prime 2}=-1$. Note that $Z_{1}^{\prime}, Z_{1}$ and $Z_{\ell_{0}}$ are distinct positive cycles with $Z_{1}^{\prime 2}=Z_{1}^{2}=Z_{\ell_{0}}^{2}=-1$, we get by Lemma 2.3 that $Z_{1}^{\prime} Z_{1}=Z_{1}^{\prime} Z_{\ell_{0}}=0$. Therefore $Z_{1}^{\prime} D=Z_{1}^{\prime} Z_{1}-Z_{1}^{\prime} Z_{\ell_{0}}=0$, which implies that $Z_{1}^{\prime 2}=D Z_{1}^{\prime}-D^{\prime} Z_{1}^{\prime}=0$, a contradiction.

Moreover we have $Z_{1}^{\prime 2}=D^{2}=-2, Z_{1}^{\prime} Z_{1}=Z_{1}^{2}=-1, Z_{1}^{\prime} Z_{\ell_{0}}=-Z_{\ell_{0}}^{2}=1$ and $Z_{1}^{\prime} Z_{i}=0$ for $i \neq 1, \ell_{0}$. This is case 1$)$.

(2) Assume that $D$ and $Z_{1}^{\prime}$ are disjoint. Since $Z_{1}=D+Z_{\ell_{0}} \geq Z_{1}^{\prime}, Z_{\ell_{0}} \geq Z_{1}^{\prime}$. By Theorem 2.2, one gets $Z_{1}^{\prime} Z_{i}=0$ for any $i<\ell_{0}$. Note that $\ell_{0}=d_{0} \geq 2$, we get $Z_{1} Z_{1}^{\prime}=0$, so $\Gamma$ is not contained in $Z_{1}^{\prime}$. Since $Z_{\ell_{0}}^{2}=-1$, we know that $\Gamma$ is the unique component of $Z_{\ell_{0}}$ with $Z_{\ell_{0}} \Gamma<0$, and for any other component $C$ of $Z_{\ell_{0}}$, we have $Z_{\ell_{0}} C=0$. From $Z_{\ell_{0}} \geq Z_{1}^{\prime}$, we see that $Z_{1}^{\prime} Z_{\ell_{0}}=0$.

In order to prove $\ell_{1}=d_{0}$, it is enough to show $Z_{1}^{\prime 2}=-1$. Consider the effective divisor $L=Z_{\ell_{0}}-Z_{1}^{\prime}-\Gamma$. $\Gamma$ is not a component of $L$. For any component $C$ of $L$, we claim that $C Z_{1}^{\prime} \leq 0$. Indeed, if $C Z_{1}^{\prime}>0$, then $C$ is not a component of $Z_{1}^{\prime}$ because $Z_{1}^{\prime}$ is a fundamental cycle. Because $C \neq \Gamma$, we know that $C$ is the exceptional curve of any other singular point $P_{i} \neq P_{1}$ of $X_{1}$, in this case, $C$ is disjoint with $Z_{1}^{\prime}$, a contradiction. Thus $C Z_{1}^{\prime} \leq 0$. Hence $L Z_{1}^{\prime}=\left(Z_{\ell_{0}}-Z_{1}^{\prime}-\Gamma\right) Z_{1}^{\prime} \leq 0$, i.e., $-Z_{1}^{\prime 2} \leq \Gamma Z_{1}^{\prime}$. 
By (7), $\Gamma \cdot \bar{\pi}^{*} \mathcal{E}_{1}=\bar{\pi}_{*} \Gamma \cdot \mathcal{E}_{1}=\bar{E}_{0} \cdot \mathcal{E}_{1}=1$, i.e., $\Gamma Z_{1}^{\prime}+\cdots+\Gamma Z_{\ell_{1}}^{\prime}=1$. Because $\Gamma$ is not a component of $Z_{i}^{\prime}$, we have $\Gamma Z_{1}^{\prime} \leq 1$. Now we get $1 \leq-Z_{1}^{\prime 2} \leq 1$, and $Z_{1}^{\prime 2}=-1$. This is case 2 ).

(3) Note that $Z_{1} Z_{\ell_{0}}=0$ and $\Gamma$ is contained in $Z_{\ell_{0}}$, we get $Z_{1} \Gamma=0$. Since $Z_{1}^{2}=-1$, we can find an irreducible component $\Gamma_{1} \neq \Gamma$ such that $\Gamma_{1} Z_{1}=-1$. Then we know that $\Gamma_{1}$ is not contained in $Z_{\ell_{0}}$.

On the other hand, suppose any singularity on $X_{1}$ is of type (2), then all of the new exceptional curves are contained in $Z_{\ell_{0}}$, which contradicts the existence of $\Gamma_{1}$. Therefore, $X_{1}$ admits at least one singular point of Type (1).

Corollary 2.3. Assume $\ell_{0}=d_{0}$. Let $\Gamma_{j}\left(j=1, \cdots, d_{0}\right)$ be the unique irreducible components such that $\Gamma_{j} Z_{j}=-1$. Then $\Gamma_{j} Z_{j+1}=1$ and $\Gamma_{j} Z_{k}=0$ $(k \neq j, j+1)$. For any other irreducible component $C \neq \Gamma_{j}$, we have $C Z_{j}=0$.

Proof. Since $Z_{j}^{2}=-1$, one can find a unique $\Gamma_{j}$ such that $\Gamma_{j} Z_{j}=-1$. By the proof of Theorem 2.4, $\bar{\pi}^{*} \bar{E}_{0}=d_{0} \Gamma_{d_{0}}$.

Since $\Gamma_{j} Z_{j}=-1$ and $Z_{j} Z_{k}=0$ (for $k \neq j$ ), $\Gamma_{j}$ does not lie in $Z_{j+1}$. Thus $\Gamma_{j} Z_{k}=0$ for $k<j$ and $\Gamma_{j} Z_{k} \geq 0$ for $k>j$. Since $\Gamma_{j} \neq \Gamma_{d_{0}}, \Gamma_{j} \cdot \bar{\pi}^{*} \mathcal{E}_{0}=0$. Thus

$$
\Gamma_{j} Z_{j}+\sum_{k>j} \Gamma_{j} Z_{k}=0
$$

Note that $\Gamma_{j} Z_{k} \geq 0$ and $\Gamma_{j} Z_{j}=-1$, we have $\Gamma_{j} Z_{j+1}=1$ and $\Gamma_{j} Z_{k}=0$ for $k>j+1$.

Let $C \neq \Gamma_{1}, \cdots, \Gamma_{d_{0}}$. If $C Z_{k}<0$ for some $k$, then $C=\Gamma_{k}$, a contradiction. So $C Z_{k} \geq 0$ for any $k$. Since $C \neq \Gamma_{d_{0}}, C \cdot \bar{\pi}^{*} \mathcal{E}_{0}=0$, i.e.,

$$
C Z_{1}+\cdots+C Z_{d_{0}}=0
$$

Hence $C Z_{k}=0$ for any $k$.

2.7. The case $\ell_{0}=d_{0}-1$.

Theorem 2.5. Suppose $\ell_{0}=d_{0}-1 \leq 2$. Suppose $P_{1}$ be any singular point of $X_{1}$. Then $-1 \leq Z_{1}^{\prime} Z_{1} \leq 0$.

1. If $\ell_{1}=d_{0}$, then $Z_{1}^{\prime} Z_{1}=\cdots=Z_{1}^{\prime} Z_{\ell_{0}}=0$ and

$$
Z_{\ell_{1}}^{\prime}<\cdots<Z_{1}^{\prime}<Z_{\ell_{0}}<\cdots<Z_{1}
$$

2. If $\ell_{1} \leq d_{0}-1$, then $Z_{1}^{\prime} Z_{1}=2-d_{0}$. Moreover, if $d_{0}=3, X_{1}$ admits at most two such singular points $P_{1}$ and $P_{2}$.

3. Assume $d_{0}=3$. $X_{1}$ has two singular points $P_{1}$ and $P_{2}$ if and only if there are two components $\Gamma_{1}$ and $\Gamma_{2}$ such that $Z_{1} \Gamma_{1}=Z_{1} \Gamma_{2}=-1$ and $\pi_{1}$ is totally ramified over $E_{0}$. In this case, $\pi_{1}^{-1}\left(p_{1}\right)=\left\{P_{1}\right\}$ and $\pi_{2}^{-1}\left(p_{2}\right)=\left\{P_{2}\right\}$, we have

$$
Z_{1}=2 Z_{2}+Z_{1}^{\prime}+Z_{1}^{\prime \prime}
$$

where $Z_{1}^{\prime}$ (resp. $Z_{1}^{\prime \prime}$ ) is the fundamental cycle of the exceptional set corresponding to $P_{1}$ (resp. $P_{2}$ ). Moreover, $Z_{1}^{\prime} \Gamma_{1}=Z_{1}^{\prime \prime} \Gamma_{2}=-3$.

Proof. If $d_{0}=2$, then $\ell_{0}=1$, i.e., $\bar{\pi}^{*} \mathcal{E}_{0}=Z_{1}$. So $Z_{1}^{\prime} Z_{1}=Z_{1}^{\prime} \cdot \bar{\pi}^{*} \mathcal{E}_{0}=0$. Everything is trivial. In what follows, we assume $d_{0}=3$.

Since $Z_{1}^{\prime} \leq Z_{1}, Z_{1}^{\prime} Z_{1} \leq 0$. From $\left(Z_{2}+Z_{1}^{\prime}\right)^{2}<0$ and $Z_{2}^{2}=-1$, we get $Z_{1}^{\prime} Z_{2} \leq 1$. Thus $Z_{1}^{\prime} Z_{1}=-Z_{1}^{\prime} Z_{2} \geq-1$. The equality implies $Z_{1}^{\prime 2} \leq-2$. 
Let $\Gamma$ be a component as in Corollary 2.2. Since $\Gamma \cdot \bar{\pi}^{*} \mathcal{E}_{1}=1, \Gamma Z_{1}^{\prime}=1$.

(1) Assume $Z_{1}^{\prime} Z_{2}=0$, i.e., $Z_{1}^{\prime} Z_{1}=0$. Let $C$ be any irreducible component of $Z_{1}^{\prime}$. Since $C \neq \Gamma, C Z_{2} \geq 0$. Hence $C Z_{2}=0$. Note that $\Gamma Z_{1}^{\prime}=1$ and $\Gamma \leq Z_{2}$. By Lemma 2.2 , it implies $Z_{1}^{\prime}<Z_{2}$. Hence $Z_{2}-\Gamma-Z_{1}^{\prime} \geq 0$.

Similar to the proof of Theorem 2.4, one can prove that $C Z_{1}^{\prime} \leq 0$ for any irreducible exceptional component $C$ of $Z_{2}-\Gamma-Z_{1}^{\prime}$. So $\left(Z_{2}-\Gamma-Z_{1}^{\prime}\right) Z_{1}^{\prime} \leq 0$. It implies that $Z_{1}^{\prime 2}=-1$, i.e., $\ell_{1}=3$. This is the case 1$)$.

(2) Assume $Z_{1}^{\prime} Z_{2}=1$, i.e., $Z_{1}^{\prime} Z_{1}=-1$. By the above discussion, $Z_{1}^{\prime 2} \leq-2$. This is the case (2).

Let $p_{1}, \cdots, p_{s}$ be the singular points in $E_{0}$ obtained in the canonical resolution. Then one can find an irreducible component $\Gamma_{i}$ in the exceptional set corresponding of $P_{i}$ such that $\Gamma_{i} Z_{1}=-1$ since $Z_{1}^{\prime} Z_{1}=-1$. So

$$
-2=Z_{1}^{2} \leq \sum_{i=1}^{s} Z_{1} \Gamma_{i} \leq-s .
$$

i.e., $s \leq 2$.

(3) Assume that $s=2$. Note that $Z_{1}^{\prime} Z_{1}=Z_{1}^{\prime \prime} Z_{1}=-1$ and $Z_{1}^{\prime} Z_{2}=Z_{1}^{\prime \prime} Z_{2}=1$. One has

$$
\left(Z_{1}-2 Z_{2}-Z_{1}^{\prime}-Z_{1}^{\prime \prime}\right)^{2}=6+Z_{1}^{\prime 2}+Z_{1}^{\prime \prime 2} \leq 0 .
$$

Since $Z_{1}^{\prime 2} \geq-3$ and $Z_{1}^{\prime \prime 2} \geq-3$, we get $Z_{1}^{\prime 2}=Z_{1}^{\prime \prime 2}=-3$ and $Z_{1}=2 Z_{2}+Z_{1}^{\prime}+Z_{1}^{\prime \prime}$. Thus $Z_{1} \geq 2 Z_{2} \geq 2 \Gamma$ and hence $\bar{\pi}^{*} \bar{E}_{0} \geq 3 \Gamma$, i.e., $\bar{\pi} * \bar{E}_{0}=3 \Gamma$, and $\pi_{1}$ is totally ramified over $E_{0}$. Since $\Gamma_{1} Z_{1}=-1$ and $\Gamma_{1} Z_{2}=1, Z_{1}^{\prime} \Gamma_{1}=Z_{1} \Gamma_{1}-2 Z_{2} \Gamma_{1}=-3$. Similarly, we have $Z_{1}^{\prime \prime} \Gamma_{2}=-3$.

Conversely, we assume that there are two components $\Gamma_{1}$ and $\Gamma_{2}$ such that $Z_{1} \Gamma_{1}=$ $Z_{1} \Gamma_{2}=-1$ and $\bar{\pi}^{*} \bar{E}_{0}=3 \Gamma$, we claim that there are two singular points $p_{1}$ and $p_{2}$ in $E_{0}$.

Indeed, $\Gamma \neq \Gamma_{1}, \Gamma_{2}$, i.e., $E_{0} \neq \bar{\pi}\left(\Gamma_{1}\right)$ and $\bar{\pi}\left(\Gamma_{2}\right)$. One can find a singular point $p_{1}$ in $E_{0}$ such that $\Gamma_{1} \leq Z_{1}^{\prime}$. If $Z_{1}^{\prime}$ also contains $\Gamma_{2}$, then $Z_{1} Z_{1}^{\prime} \leq Z_{1} \Gamma_{1}+Z_{1} \Gamma_{2}=-2$, which contradicts to (2). Hence $Z_{1}^{\prime}$ does not contain $\Gamma_{2}$ and one can find another singular point $p_{2}$ in $E_{0}$ such that $\Gamma_{2} \leq Z_{1}^{\prime \prime}$. Thus $Z_{1} Z_{1}^{\prime}=Z_{1} Z_{1}^{\prime \prime}=-1$.

2.8. The case $\ell_{0}=d_{0}=3$. In this case, we will always assume that $P_{1}$ is a singular point of $X_{1}$ of type $(3,2)$. What we are going to consider is the type of $P_{2}$.

$$
P_{2} \rightarrow P_{1} \rightarrow P_{0}
$$

Theorem 2.6. Assume that $\ell_{0}=d_{0}=3$ and $\pi_{2}$ is totally ramified over $E_{1}$. Suppose $p_{2}=E_{0} \cap E_{1} . \pi_{2}^{-1}\left(p_{2}\right)=\left\{P_{2}\right\}$. Let $\Gamma_{1}, \Gamma_{2}$ and $\Gamma_{3}$ be the irreducible components satisfying $\Gamma_{i} Z_{i}=-1$ as in Corollary 2.3. Then

1. $Z_{2}^{\prime} \Gamma_{1}=Z_{2}^{\prime} \Gamma_{2}=Z_{1}^{\prime} \Gamma_{3}=1, Z_{1}^{\prime} \Gamma_{1}=Z_{1}^{\prime} \Gamma_{2}=-1$ and $Z_{2}^{\prime} \Gamma_{3}=0$.

2. $Z_{3}$ and $Z_{2}^{\prime}$ are disjoint.

3. $Z_{2}-Z_{3}=Z_{1}^{\prime \prime}+Z_{2}^{\prime}$.

4. $F_{2}=Z_{1}^{\prime \prime}, Z_{1}^{\prime \prime} \Gamma_{2}=-3$ and $\bar{E}_{2}$ does not lie in the branch locus.

Proof. Let $D=Z_{2}-Z_{3}$.

(1) By Corollary 2.3 and $Z_{1}^{\prime}=Z_{1}-Z_{3}$, one has $Z_{1}^{\prime} \Gamma_{1}=Z_{1}^{\prime} \Gamma_{2}=-1$ and $Z_{1}^{\prime} \Gamma_{3}=1$. Since $Z_{1}^{\prime} Z_{2}^{\prime}=0$ and $Z_{1}^{\prime}>Z_{2}^{\prime}, Z_{2}^{\prime}$ does not contain $\Gamma_{k}$. From Corollary 2.2 and $E_{1}$ is totally ramified, $\bar{E}_{1} \neq \bar{\pi}\left(\Gamma_{1}\right), \bar{\pi}\left(\Gamma_{2}\right)$. Hence $\bar{\pi}^{*} \mathcal{E}_{1} \cdot \Gamma_{1}=\bar{\pi}^{*} \mathcal{E}_{1} \cdot \Gamma_{2}=0$. So $Z_{2}^{\prime} \cdot \Gamma_{1}=Z_{2}^{\prime} \cdot \Gamma_{2}=1$. Since $1=\Gamma_{3} \cdot \bar{\pi}^{*} \mathcal{E}_{1}$ and $Z_{1}^{\prime} \cdot \Gamma_{3}=1, Z_{2}^{\prime} \Gamma_{3}=0$. 
(2) Since $Z_{3}^{2}=Z_{2}^{\prime 2}=-1$, by Lemma 2.3 , either $Z_{3}$ and $Z_{2}^{\prime}$ are disjoint or $Z_{3}>Z_{2}^{\prime}$ or $Z_{3}<Z_{2}^{\prime}$. However, the last case is impossible since it implies $\Gamma_{3} \leq Z_{2}^{\prime}$. Suppose that $Z_{3}>Z_{2}^{\prime}$. Since $Z_{2}^{\prime} \Gamma_{1}=1$ and $\Gamma_{1}$ does not lie $Z_{3}, Z_{3} \Gamma_{1} \geq Z_{2}^{\prime} \Gamma_{1}=1$. which contradicts Corollary 2.3. So $Z_{3}$ and $Z_{2}^{\prime}$ are disjoint. In particular, $\Gamma_{3}$ and $Z_{2}^{\prime}$ are disjoint.

(3) From Theorem 2.5, if $Z_{1}^{\prime \prime} Z_{2}^{\prime}=0$, then $Z_{1}^{\prime \prime}<Z_{2}^{\prime}$. Since $Z_{1}^{\prime \prime} \Gamma_{3}>0, Z_{2}^{\prime} \Gamma_{3}>0$, which contradicts (2). Hence $Z_{1}^{\prime \prime} Z_{2}^{\prime}=1$ by Theorem 2.5 . Thus $Z_{1}^{\prime \prime} Z_{1}^{\prime}=-1$.

Since $Z_{1}^{\prime} \Gamma_{1}=Z_{1}^{\prime} \Gamma_{2}=Z_{1}^{\prime \prime} Z_{1}^{\prime}=-1, Z_{1}^{\prime \prime}$ contains one of $\Gamma_{1}$ and $\Gamma_{2}$. If $Z_{1}^{\prime \prime}$ contains $\Gamma_{1}$, then $Z_{3} Z_{1}^{\prime \prime}=0$ by Corollary 2.3. Moreover Corollary 2.3 implies $Z_{3} C \geq 0$ for any irreducible component $C \leq Z_{1}^{\prime \prime}$. By Lemma $2.3, Z_{3}$ and $Z_{1}^{\prime \prime}$ are disjoint, which contradicts the fact $\Gamma_{3} Z_{1}^{\prime \prime}>0$. Thus $Z_{1}^{\prime \prime}$ contains $\Gamma_{2}$. Hence $Z_{1}^{\prime \prime} Z_{3}=1, Z_{1}^{\prime \prime} Z_{2}=-1$ by Corollary 2.3. So $Z_{1}^{\prime \prime} D=-2$.

By Lemma 2.3, $Z_{2} Z_{2}^{\prime}=Z_{3} Z_{2}^{\prime}=0$, hence $D Z_{2}^{\prime}=0$. Now we obtain

$$
\left(D-Z_{1}^{\prime \prime}-Z_{2}^{\prime}\right)^{2}=3+Z_{1}^{\prime \prime 2} \geq 0 .
$$

So $D=Z_{1}^{\prime \prime}+Z_{2}^{\prime}$ and $Z_{1}^{\prime \prime 2}=-3$, i.e., $\bar{\pi}^{*} \mathcal{E}_{2}=Z_{1}^{\prime \prime}$.

(4) Since $D \Gamma_{2}=Z_{2} \Gamma_{2}-Z_{3} \Gamma_{2}=-2$ and $Z_{2}^{\prime} \cdot \Gamma_{2}=1, Z_{1}^{\prime \prime} \cdot \Gamma_{2}=-3$. Thus $\bar{\pi}_{*} \Gamma_{2} \cdot \mathcal{E}_{2}=\Gamma_{2} \cdot \bar{\pi}^{*} \mathcal{E}_{2}=\Gamma_{2} \cdot Z_{1}^{\prime \prime}=-3$, which implies $\bar{\pi}_{*} \Gamma_{2}=3 \bar{E}_{2}$. Thus $E_{2}$ is not in branch locus.

Corollary 2.4. Under the assumptions of Theorem 2.6, if $\pi_{2}$ is totally ramified over $E_{1}$, then there is a singular point $p_{3}\left(\neq p_{2}\right)$ in $E_{1}$ such that $Z_{1}^{\prime \prime \prime} \Gamma_{1}=-3$.

$$
Z_{1}^{\prime}=2 Z_{2}^{\prime}+Z_{1}^{\prime \prime}+Z_{1}^{\prime \prime \prime}
$$

Proof. It follows from Theorem 2.5 and Theorem 2.6.

2.9. (-1)-curves in the canonical resolution. Let $\tau: \widetilde{X} \rightarrow S$ be the contraction map of those $(-1)$-curves in the exceptional set of Jung's resolution $J: \widetilde{X} \rightarrow(X, P)$. We get a minimal resolution $\eta: S \rightarrow X$.

Similar to $\sigma$, we let $D_{1}, \cdots, D_{r}$ be the exceptional curves of $\tau$ and denote by $\mathcal{D}_{i}$ the total transform of $D_{i}$ in $\tilde{X}$. Then we know that

$$
\mathbb{Z} \widetilde{D}_{1}+\cdots+\mathbb{Z} \widetilde{D}_{r}=\mathbb{Z} \mathcal{D}_{1}+\cdots+\mathbb{Z} \mathcal{D}_{r}
$$

For each $i$ and $j \neq i, \mathcal{D}_{i}^{2}=-1$ and $\mathcal{D}_{i} \cdot \mathcal{D}_{j}=0$.

TheOrem 2.7. $\mathcal{D}_{1}, \cdots, \mathcal{D}_{r}$ are exactly the $(-1)$-cycles in the set $\mathcal{F u n d}(J)$ of fundamental cycles.

$$
\left\{\mathcal{D}_{1}, \cdots, \mathcal{D}_{r}\right\}=\left\{Z \in \mathcal{F} \text { und }(J) \mid p_{a}(Z)=0, Z^{2}=-1\right\}
$$

Proof. If $Z_{j}^{(i)}$ is a $(-1)$-cycle, then the curves in $Z_{j}^{(i)}$ are contracted by $\tau$. So $Z_{j}^{(i)}$ is a divisor in $\mathbb{Z} \mathcal{D}_{1}+\cdots+\mathbb{Z} \mathcal{D}_{r}$. Since $Z_{j}^{(i)} \cdot Z_{j}^{(i)}=-1$, by Lemma 2.4 , we have $Z_{j}^{(i)}=\mathcal{D}_{\ell}$ for some $\ell$.

Conversely, suppose some $\mathcal{D}_{\ell} \neq Z_{j}^{(i)}$ for any $i$ and $j$. Note that $\operatorname{Supp}\left(\bar{\pi}_{*} \mathcal{D}_{\ell}\right) \subset \mathcal{E}_{0}$. Suppose $i$ is the maximal integer such that

$$
\operatorname{Supp}\left(\bar{\pi}_{*} \mathcal{D}_{\ell}\right) \subset \mathcal{E}_{i} .
$$


Thus the strict transform of $E_{i}$ in $\bar{Y}$ must be contained in $\bar{\pi}_{*} \mathcal{D}_{\ell}$, hence $\bar{\pi}_{*} \mathcal{D}_{\ell} \cdot \mathcal{E}_{i}<0$. Thus

$$
\mathcal{D}_{\ell} Z_{1}^{(i)}+\cdots+\mathcal{D}_{\ell} Z_{\ell_{i}}^{(i)}=\mathcal{D}_{\ell} \cdot \bar{\pi}^{*} \mathcal{E}_{i}=\bar{\pi}_{*} \mathcal{D}_{\ell} \cdot \mathcal{E}_{i}<0
$$

implies $D_{\ell} Z_{j}^{(i)}<0$ for some $j$. Furthermore, by Lemma 2.3 , one has $Z_{j}^{(i)} \cdot Z_{j}^{(i)} \leq-2$ and hence $j=1$.

By assumption, the support of $\mathcal{D}_{\ell}$ is contained in $\bar{\pi}^{*} \mathcal{E}_{i}$, hence in $Z_{1}^{(i)}$, by Lemma 2.5 and our assumption, we get $\mathcal{D}_{\ell} Z_{1}^{(i)}=0$, a contradiction.

REMARK 2.1. By Theorem 2.2, $F_{i}$ has a decomposition as (12) for $i=$ $0,1, \cdots, k-1$.

$$
F_{i}=Z_{1}^{(i)}+\cdots+Z_{\ell_{i}}^{(i)}
$$

For convenience, we will also write the decomposition as follows.

$$
F_{i}=Z_{1}^{(i)}+\cdots+Z_{d_{i}}^{(i)}
$$

where

$$
Z_{1}^{(i)}>\cdots>Z_{\ell_{i}}^{(i)}>0 \quad \text { and } \quad Z_{\ell_{i}+1}^{(i)}=\cdots=Z_{d_{i}}^{(i)}=0
$$

3. Numerical invariants of double points. In this section, we will give new proofs of some results on double points $(X, P)$ by our method.

3.1. Decomposition of cycles and invariants . In this section, $d_{0}=2$. Let $\pi_{0}$ be determined by the double cover data $\left(B_{0}, \delta_{0}\right)$, i.e., $B_{0} \equiv 2 \delta_{0}$.

Denote by $m_{i}$ the multiplicity of the branch locus $B_{i}$ of $\pi_{i}$ at $p_{i}, w_{i}$ is the integral part of $m_{i} / 2$.

$$
m_{i}=\operatorname{mult}_{p_{i}}\left(B_{i}\right), \quad w_{i}=\left[\frac{m_{i}}{2}\right] .
$$

$\bar{\sigma}$ is minimal iff $m_{i} \geq 2$, equivalently $w_{i} \geq 1$ for any $i$. The double cover data $(\bar{B}, \bar{\delta})$ of $\bar{\pi}$ satisfies

$$
\bar{\delta}=\sigma^{*}\left(\delta_{0}\right)-\sum_{i=0}^{k-1} w_{i} \mathcal{E}_{i} .
$$

By the formulas for double covers (see [3], Ch.III, $\S 7$ ), we can compute the rational canonical divisor of the canonical resolution $\bar{\sigma}$.

$$
K=\sum_{i=0}^{k-1}\left(1-w_{i}\right) \cdot \bar{\pi}^{*} \mathcal{E}_{i} .
$$

Note that $w_{i} \geq 1$ for any $i$.

Lemma 3.1. For the canonical resolution $\bar{\sigma},-K$ is an effective divisor. $K \bar{E}_{s}<0$ iff $\bar{E}_{s}$ is a $(-1)$-curve.

Note that $\pi_{i}^{-1}\left(p_{i}\right)=\left\{P_{i}\right\}$, and $F_{i}=\bar{\pi}^{*} \mathcal{E}_{i}$ is connected. By Theorem 2.2, each $\bar{\pi}^{*} \mathcal{E}_{i}$ has a unique decomposition

$$
F_{i}=\bar{\pi}^{*} \mathcal{E}_{i}=Z_{1}^{(i)}+\cdots+Z_{\ell_{i}}^{(i)} .
$$

Corollary 3.1. $\ell_{i-1} \leq 2$. 
I) If $\ell_{i-1}=1$, then $\left(Z_{1}^{(i)}\right)^{2}=-2$.

II) If $\ell_{i-1}=2$, then $\left(Z_{1}^{(i)}\right)^{2}=\left(Z_{2}^{(i)}\right)^{2}=-1$, and $\bar{E}_{i-1}$ is in the branch locus.

Furthermore, $Z_{j, i}=Z_{j^{\prime}, i^{\prime}}$ iff $i=i^{\prime}$ and $j=j^{\prime}$ by Corollary 2.1.

Corollary 3.2. $K \cdot \bar{\pi}^{*} \mathcal{E}_{i}=2 w_{i}-2, p_{a}\left(\bar{\pi}^{*} \mathcal{E}_{i}\right)=w_{i}-1$ and

$$
w_{i}=p_{a}\left(Z_{1}^{(i)}\right)+\cdots+p_{a}\left(Z_{\ell_{i}}^{(i)}\right)+d_{i}-\ell_{i},
$$

Proof. Let $Z_{j}^{(i)}=0$ for $j>\ell_{i}$. By the adjunction formula and (15),

$$
\begin{aligned}
p_{a}\left(Z_{1}^{(i)}\right)+p_{a}\left(Z_{2}^{(i)}\right) & =1+\frac{1}{2}\left(\left(Z_{1}^{(i)}\right)^{2}+K Z_{1}^{(i)}\right)+1+\frac{1}{2}\left(\left(Z_{2}^{(i)}\right)^{2}+K Z_{2}^{(i)}\right) \\
& =2+\frac{1}{2}\left(\left(\bar{\pi}^{*} \mathcal{E}_{i}\right)^{2}+K \cdot \bar{\pi}^{*} \mathcal{E}_{i}\right) \\
& =2+\frac{1}{2}\left(-2-2\left(1-w_{i}\right)\right)=w_{i} .
\end{aligned}
$$

COROLlary 3.3. $Z_{1}=Z_{1}^{(0)}$ is the fundamental divisor of the canonical resolution of $(X, P)$ and $Z_{1} \geq Z_{j}^{(i)}$ for any $i$ and $j$.

Proof. By definition, $Z_{1}=Z_{1}^{(0)}$ is the fundamental cycle of the canonical resolution. Note that the support of $Z_{j}^{(i)}$ is contained in $Z_{1}$. We write $Z_{1}=Z^{\prime}+Z^{\prime \prime}$, where $Z^{\prime}$ has the same support as $Z_{j}^{(i)}$, and $Z^{\prime}$ has no common component with $Z^{\prime \prime}$. Then for any curve $\Gamma$ in $Z_{j}^{(i)}$, we have $\Gamma Z^{\prime} \leq \Gamma Z_{1} \leq 0$, by definition, $Z^{\prime} \geq Z_{j}^{(i)}$, so $Z_{1} \geq Z_{j}^{(i)}$.

3.2. A relation between the invariants. From Theorem 2.4, we have

Theorem 3.1. Assume that $\ell_{0}=2$ and $p_{1} \rightarrow p_{0}$.

I) If $\ell_{1}=1$, then $Z_{1}^{\prime}=Z_{1}-Z_{2}$. In this case,

$$
\begin{aligned}
& w_{1}=p_{a}\left(Z_{1}\right)-p_{a}\left(Z_{2}\right)+1, \\
& w_{0}=p_{a}\left(Z_{1}\right)+p_{a}\left(Z_{2}\right) .
\end{aligned}
$$

II) If $\ell_{1}=2$, then

$$
Z_{1}>Z_{2}>Z_{1}^{\prime}>Z_{2}^{\prime}
$$

and the intersection number of any two distinct cycles in this chain is zero.

In particular, exactly one point $p_{1}$ on $E_{0}$ is of type $\left.\mathrm{I}\right)$.

EXAmple 3.1 . Let $(X, P)$ be defined by $z^{2}=y\left(x^{4}+y^{6}\right)$. There are two infinitely closed singular points $p_{1}, p_{2}$ in $E_{0}$ where $p_{1}$ is of type I) and $p_{2}$ is of type II). $Z_{1}^{\prime}=$ $Z_{1}-Z_{2}$ and $Z_{1}^{\prime \prime 2}=-1$. $w_{0}=2, w_{1}=1, w_{2}=1$. 
3.3. (-1)-curves in the canonical resolution. Let $\tau: \bar{X} \rightarrow S$ be the contraction map of those $(-1)$-curves in the exceptional set of the canonical resolution. We get a minimal resolution $\eta: S \rightarrow X$.

From Theorem $1.4, \mathcal{D}_{1}, \cdots, \mathcal{D}_{r}$ are exactly the set of $(-1)$-cycles $Z_{j}^{(i)}$.

$$
\left\{\mathcal{D}_{1}, \cdots, \mathcal{D}_{r}\right\}=\left\{Z_{j}^{(i)} \mid p_{a}\left(Z_{j}^{(i)}\right)=0,\left(Z_{j}^{(i)}\right)^{2}=-1\right\}
$$

TheOREm 3.2. For any $\ell, \mathcal{D}_{\ell}=\bar{D}_{\ell}$ consists of only one irreducible $(-1)$-curve $\bar{D}_{\ell}$. Namely $\bar{D}_{1}, \cdots, \bar{D}_{r}$ are $r$ disjoint $(-1)$-curves.

Proof. From $w_{i} \geq 1$, we see that $Z_{1}^{(i)}$ can not be a $(-1)$-cycle. Suppose $\mathcal{D}_{\ell}=Z_{2}^{(i)}$ for some $i$. Since $\bar{D}_{\ell} \cdot Z_{2}^{(i)}=\bar{D}_{\ell} \cdot \mathcal{D}_{\ell}=-1$ and $\bar{D}_{\ell} \cdot Z_{1}^{(i)}=0$, we have

$$
\bar{\pi}_{*}\left(\bar{D}_{\ell}\right) \cdot \mathcal{E}_{i}=\bar{D}_{\ell} \cdot \bar{\pi}^{*}\left(\mathcal{E}_{i}\right)=\bar{D}_{\ell} \cdot\left(Z_{1}^{(i)}+Z_{2}^{(i)}\right)=-1,
$$

so $\bar{\pi}_{*}\left(\bar{D}_{\ell}\right)=\bar{E}_{i}$ and $\bar{\pi}_{*}\left(\bar{D}_{\ell}\right)$ is in the branch locus.

We will prove that $\bar{E}_{i}$ is a $(-2)$-curve, i.e., $\bar{D}_{\ell}$ is a $(-1)$-curve.

Suppose that $\bar{D}_{\ell}$ is not a $(-1)$-curve. There is another component $\bar{D}_{s}$ in $\mathcal{D}_{\ell}$ such that $\bar{D}_{\ell} \bar{D}_{s}=1$. Let $\mathcal{D}_{s}$ be the $(-1)$-cycle such that $\mathcal{D}_{s} \bar{D}_{s}=-1$. It is easy to see $\mathcal{D}_{s}<\mathcal{D}_{\ell}$ and $\bar{D}_{\ell} \mathcal{D}_{s}=1$.

We can find a singular point $p_{s}$ with a decomposition $\bar{\pi}^{*} \mathcal{E}_{s}=Z_{1, s}+Z_{2, s}$ such that $\mathcal{D}_{s}=Z_{2, s}$. Since $\mathcal{D}_{s}<\mathcal{D}_{\ell}, \mathcal{E}_{s}<\mathcal{E}_{i}$ by the choice of $\mathcal{E}_{s}$. Since $\bar{E}_{i}$ does not lie in $\mathcal{E}_{s}$, $\bar{D}_{\ell} Z_{1, s} \geq \bar{D}_{\ell} \mathcal{D}_{s}=1$. Hence $E \mathcal{E}_{s}=\bar{D}_{\ell} \bar{\pi}^{*} \mathcal{E}_{s} \geq \bar{D}_{\ell} Z_{1, s}+\bar{D}_{\ell} \mathcal{D}_{s} \geq 2$, a contradiction.

Corollary 3.4. Each $(-1)$-curve $\bar{D}_{\ell}$ comes from a singular point $\left(B_{i-1}, p_{i-1}\right)$ of type $(2 k+1 \rightarrow 2 k+1)$. Namely, $\left(B_{i-1}, p_{i-1}\right)$ is a curve singularity of multiplicity $2 k+1$, whose strict transform $\bar{B}_{i-1}$ under $\sigma_{i}$ admits only one singular point at $p_{i}=$ $\bar{B}_{i-1} \cap E_{i-1}$ with multiplicity $2 k+1$ and $E_{i-1}$ intersects $\bar{B}_{i-1}$ at $p_{i}$ transversely.

REMARK 3.1. In $[15,16]$, the singular point $p_{i-1}$ of type $(2 k+1 \rightarrow 2 k+1)$ is just the one such that $Z_{2}^{(i)}$ is a $(-1)$-curve. Thus the number of $(-1)$-curves in the exceptional set of the canonical resolution is equal to the number of singular points of the branch locus with types $(2 k+1 \rightarrow 2 k+1)$ for some positive integers $k$.

3.4. A criterion for rational double points. As an application, we obtain two well-known criteria for double points to be rational via the canonical resolution. Recall that $(X, P)$ is rational iff the fundamental cycle $Z$ of one resolution satisfies $p_{a}(Z)=0$

TheOREM 3.3. A double point $(X, P)$ is rational if and only if $w_{i}=1$ for all $i$.

Proof. We have seen that the fundamental cycle of the canonical resolution is $Z_{1}=Z_{1}^{(0)}$

Suppose $(X, P)$ is a rational double point. Then $p_{a}\left(Z_{1}\right)=0$ and $Z_{1}^{2}=-2$, so $Z_{2}=0, K Z_{1}=0$, and $w_{0}=p_{a}\left(Z_{1}\right)+p_{a}\left(Z_{2}\right)=1$. Since $Z_{1} \geq Z_{j}^{(i)}$, if $Z_{j}^{(i)} \neq 0$, then we have $0 \leq p_{a}\left(Z_{j}^{(i)}\right) \leq p_{a}\left(Z_{1}\right)=0$, so $p_{a}\left(Z_{j}^{(i)}\right)=0$. Note that $Z_{1}^{(i)} \neq 0$, we have

$$
1 \leq w_{i}=p_{a}\left(Z_{1}^{(i)}\right)+p_{a}\left(Z_{2}^{(i)}\right)=p_{a}\left(Z_{2}^{(i)}\right) \leq 1,
$$

hence $w_{i}=1$ and $Z_{2}^{(i)}=0$ for any $i$. 
Conversely, suppose $w_{i}=1$ for any $i$. By the formula (15), $K=0$.

$$
\left(Z_{j}^{(i)}\right)^{2}=\left(Z_{j}^{(i)}\right)^{2}+K Z_{j}^{(i)}=2 p_{a}\left(Z_{j}^{(i)}\right)-2 \geq-2
$$

is an even number, so $Z_{1}^{2}=-2$, which implies $Z_{2}=0$. Now we have $p_{a}\left(Z_{1}\right)=0$. By definition, $(X, P)$ is a rational double point.

Corollary 3.5. The canonical resolution $\bar{\sigma}$ of a rational double point is a minimal resolution.

According to the classification of curve singularity, the condition in the above criterion is equivalent to that the branch curve $B_{\pi}$ admits a singular point of type $\mathrm{ADE}$ at $p$ (see [3, p.78]).

4. Local invariants of singularities of multiplicity 3 . For the reader's convenience, we will introduce some basic facts on triple covers. See [12], [13] or [8] for the details. See also [1] and [14] for different methods for the study of triple covers defined by a cubic equation.

4.1. Singularities of a triple covering surface. Let $Y_{0}$ be a smooth algebraic surface over $\mathbb{C}$, and let $\pi_{0}: X_{0} \rightarrow Y_{0}$ be a normal triple cover. Then $X_{0}$ is the normalization of a surface $\Sigma$ defined by a cubic equation in a line bundle $[\mathcal{L}]$ :

$$
z^{3}+s z+t=0
$$

where $\mathcal{L}$ is an invertible sheaf, $s \in H^{0}\left(Y_{0}, \mathcal{L}^{2}\right), 0 \neq t \in H^{0}\left(Y_{0}, \mathcal{L}^{3}\right)$ and $z$ is the fiber coordinate of $[\mathcal{L}]$.

If $s=0$, then the triple cover is cyclic and everything is known (see for instance $[13$, Section 1.4] or [11]). In what follows, we assume that $s \neq 0$. Let

$$
a=\frac{4 s^{3}}{\operatorname{gcd}\left(s^{3}, t^{2}\right)}, \quad b=\frac{27 t^{2}}{\operatorname{gcd}\left(s^{3}, t^{2}\right)}, \quad c=\frac{4 s^{3}+27 t^{2}}{\operatorname{gcd}\left(s^{3}, t^{2}\right)} .
$$

Then $a, b$ and $c$ are coprime sections of an invertible sheaf such that $a+b=c$. In fact, the triple cover data $(s, t, \mathcal{L})$ is equivalent to the data $(a, b, c)$ with $a+b=c$. The latter is more convenient for the canonical resolution.

Assume that we have the following factorization of sections (according to the decompositions of their divisors)

$$
\begin{gathered}
a=4 a_{1} a_{1}^{2} a_{0}^{3}, \quad b=27 b_{1} b_{0}^{2}, \quad c=c_{1} c_{0}^{2}, \\
s=a_{1} a_{1}^{2} b_{1} a_{0}, \quad t=a_{1} a_{1}^{2} b_{1}^{2} b_{0} .
\end{gathered}
$$

where $a_{1}, a_{2}, b_{1}, c_{1}$ are square-free and are pairwise coprime.

$$
A_{i}=\operatorname{div}\left(a_{i}\right), \quad B_{i}=\operatorname{div}\left(b_{i}\right), \quad C_{i}=\operatorname{div}\left(c_{i}\right) .
$$

1. $\pi_{0}$ is totally (resp. simply) ramified over $D_{2}=A_{1}+A_{2}$ (resp. $D_{1}=B_{1}+C_{1}$ ). $R=D_{1}+2 D_{2}$ is called the branch locus.

2. Let $\pi_{0}$ be totally ramified over a singular point $p_{0}$ of $D_{1}+D_{2}$. Then $X_{0}$ is smooth over $p_{0}$ if and only if $\left(D_{1}, p_{0}\right)$ is a cusp (i.e., locally defined by $\left.y^{2}+f(x, y)^{3}=0, f(0,0)=0\right)$, and $D_{2}$ does not pass through $p_{0}$.

3. A local triple cover is Galois iff $D_{1}=0$. (This is not the case for global triple covers.)

DeFinition 4.1. A singular point of the branch locus satisfying the above condition (2) is called a good cusp of the branch locus. 
4.2. Canonical resolution. Suppose $X=X_{0} \rightarrow Y=Y_{0}$ is a triple cover. As in the double cover case, we have the following canonical resolution of the singularity $(X, P)$. We use the same notations as in the double cover case.

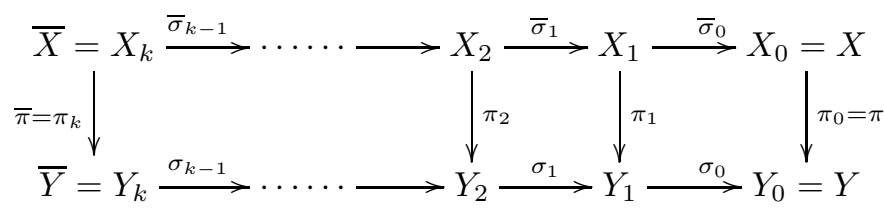

The corresponding data $\left(a^{(i+1)}, b^{(i+1)}, c^{(i+1)}\right)$ of $\pi_{i+1}$ is obtained from

$$
\left(\sigma_{i}^{*} a^{(i)}, \sigma_{i}^{*} b^{(i)}, \sigma_{i}^{*} c^{(i)}\right)
$$

by eliminating the common factors.

4.3. Local invariants of the canonical resolution. We denote by $A^{(i)}, B^{(i)}$ and $C^{(i)}$ the divisors of $a^{(i)}, b^{(i)}$ and $c^{(i)}$, respectively. Let

$$
r_{i}=\min \left\{m_{p_{i}}\left(A^{(i)}\right), m_{p_{i}}\left(B^{(i)}\right), m_{p_{i}}\left(C^{(i)}\right)\right\}
$$

where $m_{p}(D)$ is the multiplicity of a divisor $D$ at $p$. Let

$$
\begin{aligned}
m_{i} & =\left[\frac{m_{p_{i}}\left(D_{1}^{(i)}\right)}{2}\right], \\
n_{i} & = \begin{cases}m_{p_{i}}\left(D_{2}^{(i)}\right), & \text { if } r_{i} \equiv m_{p_{i}}\left(A^{(i)}\right) \quad(\bmod 3) ; \\
m_{p_{i}}\left(D_{2}^{(i)}\right)-1, & \text { otherwise. }\end{cases}
\end{aligned}
$$

Let

$$
w_{i}=m_{i}+n_{i}
$$

By some computation, we get the branch locus of $\bar{\pi}$,

$$
\left\{\begin{array}{l}
\bar{D}_{1}=\sigma^{*}\left(D_{1}\right)-2 \sum_{i=0}^{k-1} m_{i} \mathcal{E}_{i}, \\
\bar{D}_{2}=\sigma^{*}\left(D_{2}\right)-\sum_{i=0}^{k-1} n_{i} \mathcal{E}_{i} .
\end{array}\right.
$$

In the global case, we have

$$
\left\{\begin{array}{l}
p_{a}\left(\bar{D}_{1}\right)=p_{a}\left(D_{1}\right)-\sum_{i=0}^{k-1} m_{i}\left(2 m_{i}-1\right), \\
p_{a}\left(\bar{D}_{2}\right)=p_{a}\left(D_{2}\right)-\frac{1}{2} \sum_{i=0}^{k-1} n_{i}\left(n_{i}-1\right) .
\end{array}\right.
$$

5. Fundamental cycles of singularities of multiplicity 3 . The case $\pi_{i}^{-1}\left(p_{i}\right)=\left\{P_{i}, P_{i}^{\prime}\right\}$ can be reduced to the double cover case, since $d_{P_{i}}=2$ and $d_{P_{i}^{\prime}}=1$. We always assume that $d_{i-1}=3$.

We denote by $K$ the canonical divisor of the canonical resolution.

Corollary 5.1. Suppose $d_{i}=3$. Then $K \cdot \bar{\pi}^{*} \mathcal{E}_{i}=2 w_{i}-3, p_{a}\left(\bar{\pi}^{*} \mathcal{E}_{i}\right)=w_{i}-2$.

$$
w_{i}=p_{a}\left(Z_{1}^{(i)}\right)+\cdots+p_{a}\left(Z_{\ell_{i}}^{(i)}\right)+d_{i}-\ell_{i} \geq 1 .
$$


Proof. Denote by $\bar{R}_{i}$ the ramification locus over $\bar{D}_{i}$. By Hurwitz formula, $K_{\bar{X}}=$ $\bar{\pi}^{*}\left(K_{\bar{Y}}\right)+2 \bar{R}_{2}+\bar{R}_{1} \cdot K_{\bar{Y}}=\sigma^{*}\left(K_{Y}\right)+\sum_{j} \mathcal{E}_{j}$. We have

$$
\begin{aligned}
\bar{\pi}^{*} \mathcal{E}_{i} \cdot K_{\bar{X}} & =\mathcal{E}_{i} \cdot \bar{\pi}_{*}\left(K_{\bar{X}}\right)=\mathcal{E}_{i} \cdot\left(3 K_{\bar{Y}}+2 \bar{D}_{2}+\bar{D}_{1}\right) \\
& =\mathcal{E}_{i} \cdot\left(\sigma^{*}\left(3 K_{Y}+D_{1}+2 D_{2}\right)+\sum_{j}\left(3-2 m_{j}-2 n_{j}\right) \mathcal{E}_{j}\right) \\
& =2 w_{i}-3
\end{aligned}
$$

The other formulas can be obtained easily.

Corollary 5.2. Assume that $\pi$ is totally ramified over $p=\pi(P)$, i.e., $d=d_{P}=$ 3.

1. $(X, P)$ is a rational double point if and only if $w_{0}=1$ and $\ell_{0}=2$. In this case, $Z_{2}$ is a $(-1)$-cycle.

2. $(X, P)$ is a rational triple point if and only if $w_{0}=2$ and $\ell_{0}=1$.

3. If $w_{0}=1$ and $(X, P)$ is not rational, then $(X, P)$ is weakly elliptic and $\ell_{0}=3$, $p_{a}\left(Z_{1}\right)=1$. Moreover, $Z_{2}$ and $Z_{3}$ are $(-1)$-cycles.

Proof. If $(X, P)$ is rational double point, $p_{a}\left(Z_{1}\right)=0$ and $Z_{1}^{2}=-2$. From Theorem $2.2, Z_{2}^{2}=-1, Z_{3}=0$ and $w_{0}-1=p_{a}\left(Z_{1}\right)+p_{a}\left(Z_{2}\right)$. Since $Z_{2} \leq Z_{1}, p_{a}\left(Z_{2}\right)=0$ and $w_{0}=1$.

The other parts can be proved similarly.

COROLLARY 5.3. Under the assumptions in Theorem 2.6, we have

$$
\begin{aligned}
& w_{2}=p_{a}\left(Z_{2}\right)-p_{a}\left(Z_{3}\right)-p_{a}\left(Z_{2}^{\prime}\right)+2, \\
& w_{1}=p_{a}\left(Z_{1}\right)-p_{a}\left(Z_{3}\right)+p_{a}\left(Z_{2}^{\prime}\right)+1, \\
& w_{0}=p_{a}\left(Z_{1}\right)+p_{a}\left(Z_{2}\right)+p_{a}\left(Z_{3}\right) .
\end{aligned}
$$

Corollary 5.4. Under the assumptions of Corollary 2.4, we have

$$
w_{3}=p_{a}\left(Z_{1}\right)-p_{a}\left(Z_{2}\right)-p_{a}\left(Z_{2}^{\prime}\right)+2
$$

6. (-1)-curves in the canonical resolution of a triple cover. We will give a proof of the following result of [4, Lemma 5.3].

Corollary 6.1. Let $\Gamma$ be an exceptional curve in $\bar{X}$ which is contracted by $\tau$ and $E=\bar{\pi}(\Gamma)$. Let $\mathcal{D}$ be $a(-1)$-cycle such that $\mathcal{D} \Gamma=-1$ and $i$ is the maximal integer such that

$$
\operatorname{Supp}\left(\bar{\pi}_{*} \mathcal{D}\right) \subset \mathcal{E}_{i} .
$$

Then $\Gamma$ occurs in one of the following cases:

1. $E=\bar{E}_{i}$ is a (-3)-curve contained in $\bar{D}_{2}$ and $\bar{\pi}^{*} E=3 \Gamma$;

2. $E=\bar{E}_{i}$ is a (-2)-curve contained in $\bar{D}_{1}$ and $\bar{\pi}^{*} E=2 \Gamma+\bar{\Gamma}$;

3. $E$ is not a component of branch locus, but $E$ intersects with two (-3)-curves in $\bar{D}_{2}$;

4. $\pi_{i}^{-1}\left(p_{i}\right)=\left\{P_{i}, P_{i}^{\prime}\right\}$ and $\Gamma$ is a component of $F_{i}^{\prime}$. 
Proof. In what follows we always assume $\Gamma$ does not occur in case (4). By Theorem $1.4, \mathcal{D}=Z_{j}^{(i)}$ for some $j$.

Claim 1. If $E$ lies in the branch locus and $\bar{\pi}^{*} E \geq 2 \Gamma$, then $E=\bar{E}_{i}$.

Suppose that $E \neq \bar{E}_{i}$. If $\ell_{i}=2$, then $\mathcal{D}=Z_{2}^{(i)}$. Since $\Gamma Z_{1}^{(i)}=0, E \cdot \mathcal{E}_{i}=\Gamma \cdot \bar{\pi}^{*} \mathcal{E}_{i}=$ $Z_{2}^{(i)} \Gamma<0$, i.e., $E=\bar{E}_{i}$, a contradiction. Therefore $\ell_{i}=3$ and $Z_{1}^{(i)}>Z_{2}^{(i)}>Z_{3}^{(i)}>0$. If $\mathcal{D}=Z_{3}^{(i)}, E \cdot \mathcal{E}_{i}=\Gamma \cdot \bar{\pi}^{*} \mathcal{E}_{i}=Z_{3}^{(i)} \cdot \Gamma<0$, i.e., $E=\bar{E}_{i}$, a contradiction. So we have $\mathcal{D}=Z_{2}^{(i)}$. From Theorem 2.6, $E$ does not lie in the branch locus, a contradiction.

Claim 2. $\Gamma$ is a (-1)-curve if and only if $E$ lies in branch locus and $\bar{\pi}^{*} E \geq 2 \Gamma$.

If $\Gamma$ is a $(-1)$-curve, then $\Gamma=\mathcal{D}=Z_{j}^{(i)}(j \geq 2)$. By Lemma 2.3 and Lemma $2.5, \Gamma \cdot \bar{\pi}^{*} \mathcal{E}_{i}=\Gamma^{2}=-1$, hence $\Gamma$ is a component of $\bar{\pi}^{*} \bar{E}_{i} . \bar{\pi}^{*} \mathcal{E}_{i} \geq Z_{1}^{(i)}+Z_{j}^{(i)} \geq 2 \Gamma$. Namely, $E=\bar{\pi}(\Gamma)$ lies in the branch locus.

Conversely, we assume that $E$ lies in the branch locus and $\bar{\pi}^{*} E \geq 2 \Gamma$. From Claim $1, E=E_{i}$. Suppose that $\Gamma$ is not a $(-1)$-curve. There is another component $\Gamma^{\prime}$ in $\mathcal{D}$ such that $\Gamma \Gamma^{\prime}=1$. Let $\mathcal{D}^{\prime}$ be the $(-1)$-cycle such that $\mathcal{D}^{\prime} \Gamma^{\prime}=-1$. It is easy to see $\mathcal{D}^{\prime}<\mathcal{D}$ and $\Gamma \mathcal{D}^{\prime}=1$.

From Theorem 1.4, we can find a singular point $p_{s}$ with $\bar{\pi}^{*} \mathcal{E}_{s}=Z_{1}^{(s)}+Z_{2}^{(s)}+Z_{3}^{(s)}$ such that $\mathcal{D}^{\prime}=Z_{j}^{(s)}$ for some $j>1$. Since $\mathcal{D}^{\prime}<\mathcal{D}$, we have $\mathcal{E}_{s}<\mathcal{E}_{i}$ by the choice of $\mathcal{E}_{s}$. Note that $E=\bar{E}_{i}$ does not lie in $\mathcal{E}_{s}, \Gamma Z_{1}^{(s)} \geq \Gamma \mathcal{D}^{\prime}=1$. Hence $E \cdot \mathcal{E}_{s}=\Gamma \cdot \bar{\pi}^{*} \mathcal{E}_{s} \geq \Gamma Z_{1}^{(s)}+\Gamma \mathcal{D}^{\prime} \geq 2$, a contradiction.

Claim 3. If $\Gamma$ is not a (-1)-curve, then $E$ occurs in case (3).

From Claim 2 and our assumption, if $\Gamma$ is not a $(-1)$-curve, then $\ell_{i}=3, Z_{1}^{(i)}>$ $Z_{2}^{(i)}>Z_{3}^{(i)}>0$ and $\mathcal{D}=Z_{2}^{(i)}$. By Claim 2 and Corollary $2.2, \bar{E}_{i}$ is a $(-3)$-curve, $\bar{\pi}^{*} \bar{E}_{i}=3 Z_{3}^{(i)}$ and $Z_{3}^{(i)}$ is a $(-1)$-curve. Let $p_{i+1}$ be a singular point in $E_{i}\left(\subseteq Y_{i+1}\right)$ satisfying Theorem 2.4 (I) and $p_{i+2}=E_{i+1} \cap E_{i}\left(\subseteq Y_{i+2}\right)$. From Theorem 2.6 (3), $p_{a}\left(Z_{1}^{(i+2)}\right)=0$ and $Z_{2}^{(i+1)}$ is a $(-1)$-cycle. Hence $w_{i+2}=p_{a}\left(Z_{1}^{(i+2)}\right)+2=2$ and $E_{i+2}$ does not lie in the branch locus by Theorem 2.4 , and $\bar{E}_{i+1}$ is either a $(-3)$-curve in $\bar{D}_{2}$ or a $(-2)$-curve in $\bar{D}_{1}$ by Claim 2 .

Suppose that $\bar{E}_{i+1}$ is a $(-2)$-curve in $\bar{D}_{1}$. Since $\bar{E}_{i+1}$ is $(-2)$-curve, the branch locus will become smooth after blowing-up $p_{i+2}$. Furthermore, from $w_{i+2}=2$, the strict transform of the branch locus of $\pi$ in $Y_{i+2}$ is smooth at $p_{i+2}$ and does not tangent to $E_{i}, E_{i+1}$. Thus $p_{i}$ is a good cusp, namely, $X_{i}$ is smooth over $p_{i}$, which contracts our assumption. So $\bar{E}_{i+1}$ is a $(-3)$-curve over which $\bar{\pi}$ is totally ramified. $w_{i+2}=2$ implies that the strict transform of the branch locus of $\pi_{i+2}$ in $Y_{i+2}$ does not pass through $p_{i+2}$. Therefore $\Gamma=\bar{\pi}^{*} E_{i+2}$ meets exactly $E_{i+1}$ and $E_{i}$.

7. A criterion for rational singularities of multiplicity two or three - [2] listed all rational triple points associated with the Dynkin graphs which are denoted by $A_{n, m, k}, B_{n, m}, C_{n, m}, D_{n, 5}, E_{6,0}, E_{7,0}, E_{0,7}, F_{n, 6}, G_{n, 0}$ in [5],

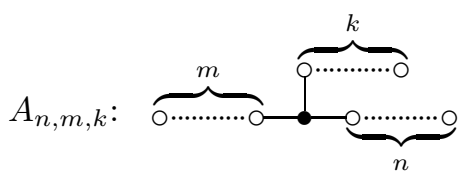

$B_{m, n}:$

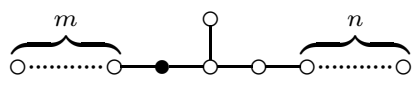




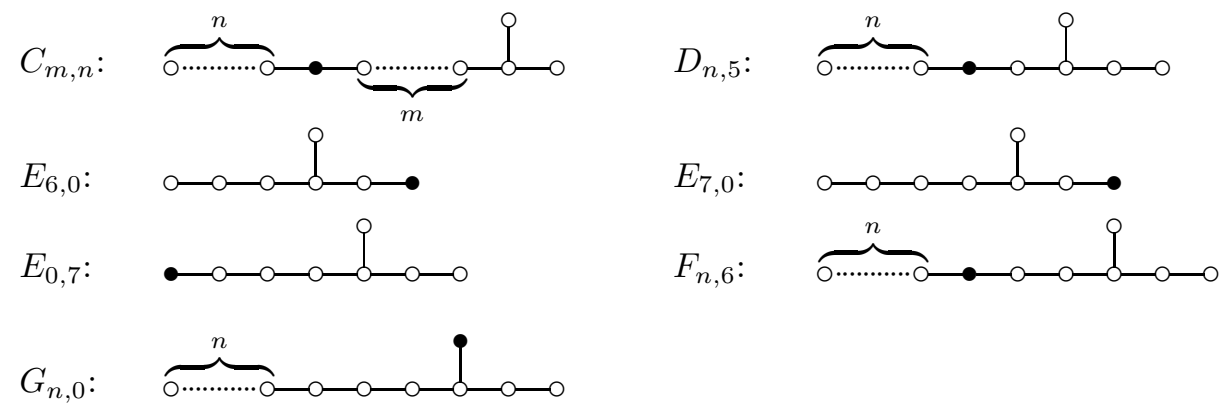

where $\circ$ is a $(-2)$-curve, $\bullet$ is a $(-3)$-curve.

THEOREM 7.1. Suppose the surface singularity $(X, P) \rightarrow(Y, p)$ is a finite cover of degree $d=2$ or 3 totally ramified over $p$. Let $P=P_{0}, P_{1}, \cdots, P_{k-1}$ be the infinitely near singular points obtained in the canonical resolution. Then

1. $w_{i} \geq 1$ for $i=0,1, \cdots, k-1$.

2. $(X, P)$ is a rational singular point iff $w_{i} \leq d-1$ for any $i$.

3. If $(X, P)$ is rational, then the multiplicity of $(X, P)$ is $w_{0}+1$.

Proof. (1) (3) follows from Corollary 5.1 and Corollary 5.2.

(2) Suppose $(X, P)$ is a rational singular point. We have seen that $Z_{1}=Z_{1}^{(0)}$ is the fundamental cycle of the canonical resolution, thus $p_{a}\left(Z_{1}\right)=0$. Because $Z_{1} \geq Z_{j}^{(i)}>0$ for $j \leq \ell_{i}$, we have $p_{a}\left(Z_{j}^{(i)}\right)=0$ for all $j \leq \ell_{i}$. Since $\ell_{i} \geq 1$ and $d_{i} \leq d$, we obtain

$$
\begin{aligned}
w_{i} & =p_{a}\left(Z_{1}^{(i)}\right)+\cdots+p_{a}\left(Z_{\ell_{i}}^{(i)}\right)+d_{i}-\ell_{i} \\
& =d_{i}-\ell_{i} \leq d-1
\end{aligned}
$$

Conversely, suppose $w_{i} \leq d-1$ for any $i$. If $d=2$, then (2) is true by Theorem 3.3. In what follows, we assume $d=3$.

Claim $1 . \ell_{0} \leq 2$.

Suppose that $\ell_{0}=3$, i.e., $Z_{3} \neq 0$. Since $w_{0} \leq 2$, by Corollary 5.1 , we have $p_{a}\left(Z_{3}\right)=0$. Let $p_{1}$ be a singular point in $E_{0}\left(\subseteq Y_{1}\right)$ satisfying Theorem 2.4 (I) and $p_{2}=E_{2} \cap E_{1}\left(\subseteq Y_{2}\right)$. From $(22)$,

$$
4 \geq w_{1}+w_{2}=p_{a}\left(Z_{1}\right)+p_{a}\left(Z_{2}\right)+3
$$

Hence $p_{a}\left(Z_{1}\right)=1, w_{1}=w_{2}=2, Z_{2}$ and $Z_{3}$ are $(-1)$-cycles. By the proof of Corollary 6.1 , the cover is totally ramified over $E_{2}$. By Corollary 5.4 , there is another singular point $p_{3}$ in $E_{2}\left(\subseteq Y_{2}\right)$. Since $w_{1}=2,1=p_{a}\left(Z_{1}^{\prime}\right)+p_{a}\left(Z_{2}^{\prime}\right)$, hence $p_{a}\left(Z_{1}^{\prime}\right)=1$ and $p_{a}\left(Z_{2}^{\prime}\right)=0$. By $(23)$, we have $w_{3}=3$, a contradiction.

Claim 2. If $w_{0}=2$, then $\ell_{0}=1$, hence $\bar{\pi}^{*} \mathcal{E}_{0}=Z_{1}$ and $p_{a}\left(Z_{1}\right)=0$, i.e., $(X, P)$ is a rational triple point.

Suppose that $\ell_{0}=2$, i.e., $Z_{2} \neq 0$. Then $1=w_{0}-1=p_{a}\left(Z_{1}\right)+p_{a}\left(Z_{2}\right)$, hence $p_{a}\left(Z_{1}\right)=1, p_{a}\left(Z_{2}\right)=0$. By Corollary $6.1, \bar{E}_{0}$ is either a $(-3)$-curve in $\bar{D}_{2}$ or a $(-2)$ curve in $\bar{D}_{1}$. Hence, if the cover is totally ramified over $\bar{E}_{1}$, then there are exactly two proximate singular points $p_{1}$ and $p_{2}$ on $E_{0}$. Otherwise, there is a unique proximate singular point on $\bar{E}_{1}$. 
From

$$
p_{a}\left(\bar{\pi}^{*} \mathcal{E}_{0}\right)=p_{a}\left(\bar{\pi}^{*} \bar{E}_{0}\right)+\sum_{p_{i} \rightarrow p} w_{i}
$$

we have

$$
w_{0}= \begin{cases}-1+\sum_{p_{i} \rightarrow p}\left(w_{i}-1\right), & \bar{\pi} \text { is totally ramified over } \bar{E}_{0}, \\ -\frac{1}{2}+\sum_{p_{i} \rightarrow p}\left(w_{i}-\frac{1}{2}\right), & \bar{\pi} \text { is simple ramified over } \bar{E}_{0},\end{cases}
$$

where $p_{i} \rightarrow p$ runs over all proximate singular points. By the above discussion, one has

$$
w_{0}= \begin{cases}w_{1}+w_{2}-3, & \bar{\pi} \text { is totally ramified over } \bar{E}_{0}, \\ w_{1}-1, & \bar{\pi} \text { is simple ramified over } \bar{E}_{0} .\end{cases}
$$

Since $w_{1}, w_{2} \leq 2$, the above equality implies $w_{0} \leq 1$, a contradiction.

Up to now, we complete the proof.

Acknowledgements. The authors would like to thank Prof. Rong Du for his discussions. They express their appreciation to the referee for very useful suggestions for the correction of the original manuscript.

\section{REFERENCES}

[1] T. Ashikaga, Normal two-dimensional hypersurface triple points and the Horikawa type resolution, Tohoku Math. J., 44:2 (1992), pp. 177-200.

[2] M. Artin, On isolated rational singularities of surfaces, Amer. J. Math., 88 (1966), pp. 129136.

[3] W. Barth, K. Hulek, C. Peters, A. Van de Ven, and A. Van de Ven, Compact Complex Surfaces, Second Edition, Springer-Verlag, Berlin, 2004.

[4] Z. Chen And S.-L. TAN, Upper bounds on the slope of a genus 3 fiberation, in Recent Progress on Some Problems in Several Complex Variables and Partial Differential Equations, Contemp. Math., 400 (2006), pp. 65-88

[5] Z. Chen, S.-L. TAN, R. Du, AND F. Yu, Cubic equations of rational triple points of dimension two, Proceedings of the International Conference on Complex Geometry and Related Fields, pp. 63-76, AMS/IP Stud. Adv. Math., 39, Amer. Math. Soc., Providence, RI, 2007.

[6] P. Griffiths and J. Harris, Principles of Algebraic Geometry, Wiley-Interscience, John Wiley \& Sons, Inc., 1978.

[7] J. Kollàr, Complex algebraic geometry, IAS/Park City mathematics series, ISSN 1079-5634; Vol. 3, 1997.

[8] J. Milnor, Singular points of complex hypersurfaces, Ann. Math. Studies, Vol. 61, Princeton University Press, Princeton, N. J., 1968.

[9] R. Miranda, Triple covers in algebraic geometry, Amer. J. Math., 107 (1985), pp. 1123-1158.

[10] P. Popescu-PAmpu, Introduction to Jung's method of resolution of singularities, Contemp. Math., 538 (2011), pp. 401-432.

[11] S.-L. TAN, Galois triple covers of surfaces, Science in China (Series A), 34:8 (1991), pp. 935942.

[12] S.-L. TAN, Integral closure of a cubic extension and applications, Proc. Amer. Math. Soc., 129:9 (2001), pp. 2553-2562.

[13] S.-L. TAN, Triple covers on smooth algebraic varieties, Stud. Adv. Math., 29 (2002), pp. 143164.

[14] H. Tokunaga, Two remarks on non-Galois triple coverings, Mem. Fac. Sci. Kochi Univ. Ser. A Math., 13 (1992), pp. 19-33.

[15] G. XIAO, Surfaces fibrées en courbes de genre deux, Lect. Notes in Math., 1137 (1985) SpringerVerlag.

[16] G. XIAO, The fibrations of algbraic surfaces, Shanghai Scientific \& Technical Publishers, 1992 (in Chinese).

[17] S. S.-T. YAU, On maximally elliptic singularities, Trans. Amer. Math. Soc, 257 (1980), pp. 269329. 